\title{
Between Poisson and GUE statistics: Role of the Breit-Wigner width
}

\author{
Klaus M. Frahm ${ }^{1}$, Thomas Guhr ${ }^{2}$ and Axel Müller-Groeling ${ }^{2}$ \\ ${ }^{1}$ Laboratoire de Physique Quantique, UMR 5626 IRSAMC, Université Paul Sabatier, F-31062 Toulouse, France \\ ${ }^{2}$ Max-Planck-Institut für Kernphysik, Postfach 103980, D-69029 Heidelberg, Germany
}

(October 6, 2018)

\begin{abstract}
We consider the spectral statistics of the superposition of a random diagonal matrix and a GUE matrix. By means of two alternative superanalytic approaches, the coset method and the graded eigenvalue method, we derive the two-level correlation function $X_{2}(r)$ and the number variance $\Sigma^{2}(r)$. The graded eigenvalue approach leads to an expression for $X_{2}(r)$ which is valid for all values of the parameter $\lambda$ governing the strength of the GUE admixture on the unfolded scale. A new twofold integration representation is found which can be easily evaluated numerically. For $\lambda \gg 1$ the Breit-Wigner width $\Gamma_{1}$ measured in units of the mean level spacing $D$ is much larger than unity. In this limit, closed analytical expression for $X_{2}(r)$ and $\Sigma^{2}(r)$ can be derived by (i) evaluating the double integral perturbatively or (ii) an ab initio perturbative calculation employing the coset method. The instructive comparison between both approaches reveals that random fluctuations of $\Gamma_{1}$ manifest themselves in modifications of the spectral statistics. The energy scale which determines the deviation of the statistical properties from GUE behavior is given by $\sqrt{\Gamma_{1}}$. This is rigorously shown and discussed in great detail. The Breit-Wigner $\Gamma_{1}$ width itself governs the approach to the Poisson limit for $r \rightarrow \infty$. Our analytical findings are confirmed by numerical simulations of an ensemble of $500 \times 500$ matrices, which demonstrate the universal validity of our results after proper unfolding.
\end{abstract}

PACS numbers: $05.40 .+\mathrm{j}, 05.45 .+\mathrm{b}, 72.15 . \mathrm{Rn}$

\section{INTRODUCTION}

One of the archetypical problems in quantum mechanics consists of calculating (certain properties of) the eigenvalue spectrum of a diagonal operator and a superimposed non-diagonal one. Little can be said in general about this problem. In our paper, we focus on the particular case where the matrix representations $H_{0}$ and $H_{1}$ of the above operators can be taken from the Poisson and the Gaussian Unitary Ensemble] (GUE), respectively,

$$
H=H_{0}+\alpha H_{1},
$$

with $\alpha$ some strength parameter. The Poisson Ensemble is constructed from all those matrices whose eigenvalues are independent random numbers with identical, and largely arbitrary, distribution function. Due to the rotational invariance of the GUE, it suffices to consider only diagonal matrices $H_{0}$ of the Poisson type. The random matrix model (1.1) should provide an adequate description for numerous transition phenomena from regular to chaotic fluctuation properties in atomic, nuclear, and condensed matter physics, as well as in quantum chaology (for a review see Ref. 2).

Nuclear physics provides an important example. In heavy ion reactions, fast rotating compound nuclei are produced. The rotation is a collective motion of all nucleons which is often accompanied by single particle excitations. Thus the total Hamiltonian can be modeled as a sum of two contributions, a regular one, $H_{0}$, describing the collective motion, and a stochastic one, $H_{1}$, describing the influence of the single particle excitations. This scenario was studied numerically in Ref. 3 for the cases that $H_{0}$ is drawn from a Poisson or a harmonic oscillator ensemble. Using Efetov's supersymmetry method 4 , the qualitative behavior of the two-level correlations was also discussed analytically in Ref. 3 .

As a further important example we mention the problem of two interacting particles in a random potential introduced by Shepelyanskyt. He predicted that two particles in a one-dimensional disorfered chain can be extended on a scale $L_{2}$ far exceeding the one-particle localization length $L_{1}$. Subsequent work 0 quickly led to a definite confirmation and better understanding of this phenomenon. One possible approach 6 to this problem is to construct an effective Hamiltonian by diagonalizing the noninteracting part of the two-electron problem and expressing the microscopic Hamiltonian in the basis of two-electron product states. The resulting representation consists of a diagonal contribution containing the eigenvalues of the noninteracting problem, and an off-diagonal contribution originating from the interaction operator. With the crucial assumption that both the diagonal and the off-diagonal matrix elements can be chosen to be random variables we arrive at the above random matrix model (for system sizes $L$ of the order of $L_{1}$ ). In the regime $L>L_{1}$ the effective Hamiltonian has been studied in some detail10 12. Imry has 
shown that the enhancement factor $L_{2} / L_{1}$ is given by the "Breit-Wigner width" or "two-particle Thouless energy" $\Gamma_{2}$ measured in units of the two-particle level spacing $D_{2}, L_{2} / L_{1}=\Gamma_{2}$. This raised, among other things, the question how to identify $\Gamma_{2}$ in typical spectral observables like the number variance $\Sigma^{2}(r)$ of the random matrix model (1.1) considered here13. This problem, and some surprises which we encountered while studying it, have been our original motivation in this project.

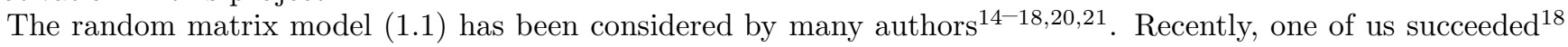
in deriving exact integral representations for the spectral $k$-point functions of the Hamiltonian (1.1) To this end,

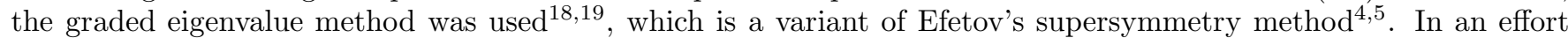
to discuss certain approximations from these integral representations, two of the present authors derived20 a closed expression for the spectral two-point function $X_{2}(r)$ by means of a special kind of saddle-point approximation. This led to the surprising observation that the energy scale at which $X_{2}(r)$ and the number variance $\Sigma^{2}(r)$ deviate from random matrix behavior is given by $\sqrt{\Gamma_{1}}$ and not by $\Gamma_{1}$. Here, $\Gamma_{1}$ is the Breit-Wigner (or spreading) width induced by the perturbation $\alpha H_{1}$ and corresponds to the quantity $\Gamma_{2}$ in the above example. Both $\Gamma_{1}$ and $r$ are measured in units of the mean level spacing $D$. This result was later confirmed in a perturbative calculation 21 of the two-point correlator $X_{2}(r)$. The saddle-point approximation employed in Ref. 20 was limited to certain situations and does not include cases in which the energy separations $r$ is much larger than the Breit-Wigner width $\Gamma_{1}$.

The purpose of the present paper is threefold. First, we derive, avoiding the above-mentioned saddle-point expression, a relatively simple exact expression for $X_{2}(r)$ for all $r$ and all relative strengths of $H_{0}$ and $H_{1}$. For $\Gamma_{1} \gg 1$ this leads to very compact analytical formulas for both $X_{2}(r)$ and $\Sigma^{2}(r)$. The two scales $\sqrt{\Gamma_{1}}$ and $\Gamma_{1}$ can be identified and interpreted. Second, we compare both variants of the supersymmetry formalism, the graded eigenvalue method and the coset method, by deriving our results independently for both methods. This sheds some light on the relation between these approaches. Furthermore our comparison elucidates the role played by statistical fluctuations of the Breit-Wigner width. Third, we confirm our result with the help of rather extensive numerical simulations of the spectral properties of the Hamiltonian (1.1).

Our paper is organized accordingly. Following this introduction, Sec. II deals with the graded eigenvalue method and the improved treatment of the integral representations derived in Ref. 18. In Sec. III the coset method is invoked to essentially re-derive the results of Sec. III. We have tried to keep both sections reasonably self-contained. Readers who are completely unfamiliar with the supersymmetry formalism should, however, consult the introductory literature

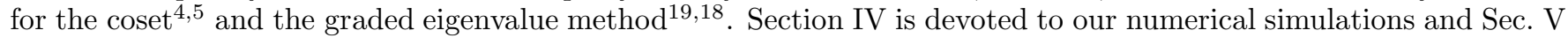
contains a summary and the discussion.

\section{GRADED EIGENVALUE METHOD}

In a first parf Sec. IIA, we introduce some basic terminology and briefly recapitulate the derivation of the integral representations 18 of the $k$-level correlation functions for the convenience of the reader. In Sec. II B the integral representation for the two-point function is further transformed to derive a relatively simple and, most importantly, tractable expression for the level-level correlator. Finally, Sec. II C is devoted to a perturbative evaluation of this expression in the limit where the Breit-Wigner width is much larger than the level spacing.

\section{A. Integral representation for spectral correlation functions}

The $k$-level correlation functions for the Hamiltonian $H$ in Eq. (1.1) characterize the spectral statistics completely and are defined by

$$
R_{k}\left(x_{1}, \ldots, x_{k}, \alpha\right)=\frac{1}{\pi^{k}} \int P_{N}(H) \prod_{p=1}^{k} \operatorname{Im} \operatorname{tr} \frac{1}{x_{p}^{-}-H} d[H] .
$$

The probability of finding $k$ energy eigenvalues in infinitesimal intervals $d x_{i}$ around $x_{i}(i=1, \ldots, k)$ is given by $R_{k}\left(x_{1}, \ldots, x_{k}\right) d x_{1} \ldots d x_{k}$. For the case considered in this paper the probability distribution function $P_{N}(H)$, which depends explicitly on the dimension $N$ of the matrices $H$, is given by the product

$$
P_{N}(H)=P_{N}^{(0)}\left(H_{0}\right) P_{N}^{(1)}\left(H_{1}\right)
$$

with 


$$
\begin{aligned}
& P_{N}^{(0)}\left(H_{0}\right)=\prod_{n=1}^{N} p^{(0)}\left(H_{0}^{n n}\right) \prod_{n>m} \delta\left(\operatorname{Re} H_{0}^{n m}\right) \delta\left(\operatorname{Im} H_{0}^{n m}\right) \\
& P_{N}^{(1)}\left(H_{1}\right)=\frac{2^{N(N-1) / 2}}{\pi^{N^{2} / 2}} \exp \left(-\operatorname{tr}\left(H_{1}^{2}\right)\right) .
\end{aligned}
$$

The function $p^{(0)}\left(H_{0}^{n n}\right)$ is smooth but otherwise arbitrary. We introduce the modified $k$-point correlators $\hat{R}_{k}\left(x_{1}, \ldots, x_{k}\right)$, which are obtained by omitting the projection onto the imaginary part in Eq. (2.1). The original quantities can be reconstructed by appropriate linear combinations of the $\hat{R}_{k}$. To perform the ensemble average we write the modified correlators in terms of a supersymmetric normalized generating functional,

$$
\hat{R}_{k}\left(x_{1}, \ldots, x_{k}, \alpha\right)=\left.\frac{1}{(2 \pi)^{k}} \frac{\partial^{k}}{\partial J_{1} \ldots \partial J_{k}} Z_{k}(x+J, \alpha)\right|_{J=0}
$$

where the energies and source variables form diagonal $2 k \times 2 k$ matrices according to $x=\operatorname{diag}\left(x_{1}, x_{1}, \ldots, x_{k}, x_{k}\right)$ and $J=\operatorname{diag}\left(-J_{1}, J_{1}, \ldots,-J_{k}, J_{k}\right)$, respectively. The averaged functional takes the form

$$
Z_{k}(x+J, \alpha)=\int d\left[H_{0}\right] P_{N}^{(0)}\left(H_{0}\right) \int d[\sigma] Q_{k}(\sigma, \alpha) \operatorname{detg}^{-1}\left[\left(x^{ \pm}+J-\sigma\right) \otimes 1_{N}-1_{2 k} \otimes H_{0}\right],
$$

where $\sigma$ is a $2 k \times 2 k$ Hermitean supermatrix and $Q_{k}(\sigma, \alpha)=2^{k(k-1)} \exp \left(-\operatorname{trg} \sigma^{2} / \alpha^{2}\right)$ is a normalized graded probability density. The subsequent steps can be summarized as follows 19 . The matrix $x+J$ is shifted from the graded determinant to the graded probability density and the supermatrix $\sigma$ is diagonalized according to $\sigma=u^{-1} s u$, where $s=\operatorname{diag}\left(s_{11}, i s_{12}, \ldots, s_{k 1}, i s_{k 2}\right)$. The volume element can be rewritten as $d[\sigma]=B_{k}^{2}(s) d[s] d \mu(u)$ with $B_{k}(s)$ the Jacobian (called Berezinian in this case) of the transformation. The non-trivial integration over the unitary diagonalizing supergroup with its Haar measure $d \mu(u)$ is the central step in the graded eigenvalue method and can be performed with the supersymmetric extension of the Harish-Chandra Itzykson Zuber integral. Collecting everything we arrive at

$$
\begin{aligned}
Z_{k}(x+J, \alpha) & =1-\eta(x+J)+\frac{1}{B_{k}(x+J)} \int G_{k}(s-x-J, \alpha) Z_{k}^{(0)}(s) B_{k}(s) d[s], \\
Z_{k}^{(0)}(x+J) & =\int d\left[H_{0}\right] P_{N}^{(0)}\left(H_{0}\right) \operatorname{detg}^{-1}\left[\left(x^{ \pm}+J\right) \otimes 1_{N}-1_{2 k} \otimes H_{0}\right],
\end{aligned}
$$

where the kernel resulting from the group integration is Gaussian and given by

$$
G_{k}(s-x, \alpha)=\frac{1}{\left(\pi \alpha^{2}\right)^{k}} \exp \left(-\frac{1}{\alpha^{2}} \operatorname{trg}(s-x)^{2}\right) .
$$

The distribution $1-\eta(x+J)$ in Eq. (2.6) ensures the normalization $Z_{k}(x, \alpha)=1$ at $J=0$. We mention in passing that the generating function $Z_{k}(x+J, \alpha)$ satisfies an exact diffusion equation in the curved space of the eigenvalues of Hermitean supermatrices. Here, $t=\alpha^{2} / 2$ is the diffusion time and the generating function $Z_{k}^{(0)}(x+J)$ serves as the initial condition. This diffusion is the supersymmetric analogue 18,22 for the diffusion of the probability distribution function in the space of ordinary matrices, which is equivalent to Dyson's Brownian. Performing the source term derivatives in Eq. (2.4) we find

$$
R_{k}\left(x_{1}, \ldots, x_{k}, \alpha\right)=\frac{(-1)^{k}}{\pi^{k}} \int G_{k}(s-x, \alpha) \Im Z_{k}^{(0)}(s) B_{k}(s) d[s],
$$

where the symbol $\Im$ indicates the above-mentioned proper linear combination of terms. In order to arrive at truly universal quantities we have to measure all energies in units of the mean level spacing $D$. This leads to the definitions $\xi_{p}=x_{p} / D, \lambda=\alpha / D, X_{k}\left(\xi_{1}, \ldots, \xi_{k}, \lambda\right)=\lim _{N \rightarrow \infty} D^{k} R_{k}\left(x_{1}, \ldots, x_{k}, \alpha\right)$, and $z_{k}^{(0)}=\lim _{N \rightarrow \infty} Z_{k}^{(0)}(D s)$. The level correlation functions $X_{k}$ defined in this way are translation invariant over the spectrum. They can be expressed as

$$
X_{k}\left(\xi_{1}, \ldots, \xi_{k}, \lambda\right)=\frac{(-1)^{k}}{\pi^{k}} \int G_{k}(s-\xi, \lambda) \Im z_{k}^{(0)}(s) B_{k}(s) d[s]
$$

after the redefinition $s \rightarrow s / D$. We notice the very similar structure of the integral representations (2.8) and (2.9). This is so because the above mentioned diffusion in superspace is, apart from the initial conditions, the same on all 
scales, in contrast to the diffusion in ordinary space which is equivalent to Dyson's Brownian. The two-point function $X_{2}$, to which we restrict our attention henceforth, depends on $r=\xi_{1}-\xi_{2}$ only and we can perform two of the four integrations in Eq. (2.9) due to translational invariance. This leads to the integral representation 18

$$
X_{2}(r, \lambda)=-\frac{8}{\pi^{3} \lambda^{2}} \int_{-\infty}^{\infty} \int_{-\infty}^{\infty} \exp \left(-\frac{1}{2 \lambda^{2}}\left(t_{1}^{2}+t_{2}^{2}\right)\right) \sinh \frac{r t_{1}}{\lambda^{2}} \sin \frac{r t_{2}}{\lambda^{2}} \frac{t_{1} t_{2}}{\left(t_{1}^{2}+t_{2}^{2}\right)^{2}} \Im z_{2}^{(0)}\left(t_{1}, t_{2}\right) d t_{1} d t_{2}
$$

with $t_{1}=s_{11}-s_{21}$ and $t_{2}=s_{12}-s_{22}$. The initial condition $z_{2}^{(0)}$ is still arbitrary. For the case of Poisson regularity we have

$$
\Im z_{2}^{(0)}\left(t_{1}, t_{2}\right)=\frac{1}{2} \operatorname{Re}\left(\exp \left(-i \pi \frac{t_{1}^{2}+t_{2}^{2}}{2 t_{1}^{-}}-1\right)\right) .
$$

Equation (2.10) is exact but, unfortunately, difficult to evaluate as it stands. In the following subsection, we derive a more convenient formulation of Eq. (2.10).

\section{B. Simplification of the two-point level correlation function $X_{2}(r, \lambda)$}

As explained in the introduction, a previous efforten to extract physical information from Eq. (2.10) led to the discovery that the energy scale at which $X_{2}(r, \lambda)$ deviates from random matrix behavior is linear in $\lambda$. For very general reasons, however, the Breit-Wigner width is always quadratic in the strength of the perturbing matrix elements, hence $\Gamma_{1} \propto \lambda^{2}$. This led to the conclusion that $\sqrt{\Gamma_{1}}$ is the important energy scale. The approximation used to derive this result was, however, not valid for $r \gg \Gamma_{1}$. Here, we avoid the saddle-point approximation proposed in Ref. 20 and proceed as follows. We introduce the new variables $x_{j}=t_{j} /\left(\pi \lambda^{2}\right)$ and the important abbreviations

$$
c=\frac{1}{\pi^{2} \lambda^{2}}, \quad \kappa=\frac{r}{\pi \lambda^{2}} .
$$

It is instructive to note that $\pi \lambda^{2}$ is actually the Breit-Wigner width measured in units of the level spacing, $\pi \lambda^{2}=\Gamma_{1}$. This can be deduced from the local density of states for the model studied here2 and will become more obvious when we discuss the coset method in the next section. With the above notation we find

$$
X_{2}(r, \lambda) \equiv X_{2}(\kappa, c)=\tilde{X}_{2}(\kappa, c)+\tilde{X}_{2}(-\kappa, c)+\kappa^{2}
$$

where

$$
\begin{aligned}
\tilde{X}_{2}(\kappa, c) & =\frac{2 i c}{\pi} \int_{-\infty}^{\infty} \int_{-\infty}^{\infty} d x_{1} d x_{2} \frac{x_{1} x_{2}}{\left(x_{1}^{2}+x_{2}^{2}\right)^{2}} \exp \left(-\mathcal{L}\left(x_{1}, x_{2}\right)\right) \\
\mathcal{L}\left(x_{1}, x_{2}\right) & =\frac{1}{2 c}\left[\frac{x_{1}}{x_{1}+i}\left(x_{1}+i+\kappa\right)^{2}+\frac{x_{1}+i}{x_{1}^{+}}\left(x_{2}+i \kappa \frac{x_{1}}{x_{1}+i}\right)^{2}\right] .
\end{aligned}
$$

To reduce the order of the pre-exponential singularity we perform an integration by parts using $2 x_{2} /\left(x_{1}^{2}+x_{2}^{2}\right)^{2}=$ $-\partial_{x_{2}}\left(x_{1}^{2}+x_{2}^{2}\right)^{-1}$. With the additional transformation $x_{2}=y_{2} x_{1} /\left(x_{1}+i\right)$ Eq. (2.14) takes the form

$$
\begin{aligned}
\tilde{X}_{2}(\kappa, c) & =-\frac{i}{\pi} \int_{-\infty}^{\infty} \int_{-\infty}^{\infty} d x_{1} d y_{2} \frac{\left(x_{1}+i\right)\left(y_{2}+i \kappa\right)}{\left(x_{1}+i\right)^{2}+y_{2}^{2}} \exp \left(-\tilde{\mathcal{L}}\left(x_{1}, y_{2}\right)\right) \\
\tilde{\mathcal{L}}\left(x_{1}, y_{2}\right) & =\frac{1}{2 c} \frac{x_{1}}{x_{1}+i}\left[\left(x_{1}+i+\kappa\right)^{2}+\left(y_{2}+i \kappa\right)^{2}\right]
\end{aligned}
$$

Now, our strategy is to eliminate the imaginary contributions to the squares in $\tilde{\mathcal{L}}\left(x_{1}, y_{2}\right)$. To this end we perform two shifts, namely $y_{1}=x_{1}+i$ and $u_{2}=y_{2}+i \kappa$. This amounts to moving the integration contour from the real axis to the lines $\operatorname{Im}\left(x_{1}\right)=-1$ and $\operatorname{Im}\left(y_{2}\right)=-\kappa$, respectively, see Fig. 11. The integral (2.15) remains unchanged under these shifts unless the integrand exhibits singularities in the region between the old and the new integration contours. With the help of Fig. 1 the reader can easily convince himself that such singularities do indeed exist in our case. They give rise to two different residuum contributions $R_{1}$ and $R_{2}$. We therefore arrive at 


$$
\begin{aligned}
\tilde{X}_{2}(\kappa, c) & =-\frac{i}{\pi} \int_{-\infty}^{\infty} \int_{-\infty}^{\infty} d y_{1} d u_{2} \frac{y_{1} u_{2}}{y_{1}^{2}+\left(u_{2}-i \kappa\right)^{2}} \exp \left(-\tilde{\mathcal{L}}\left(y_{1}-i, u_{2}-i \kappa\right)\right)-R_{1}-R_{2} \\
R_{1} & =\int_{-1}^{0} d y_{2}\left(y_{2}+i \kappa\right)+\int_{0}^{1} d y_{2}\left(y_{2}+i \kappa\right) \exp \left(2 i \frac{\kappa}{c}\left(1-y_{2}\right)\right) \\
R_{2} & =\int_{0}^{|\kappa|} d y_{1}\left(\frac{\kappa}{|\kappa|} y_{1}-\kappa\right) \exp \left(-\left(y_{1}-i\right) \frac{\kappa+|\kappa|}{c}\right)+\int_{-|\kappa|}^{0} d y_{1}\left(\frac{\kappa}{|\kappa|} y_{1}+\kappa\right) \exp \left(-\left(y_{1}-i\right) \frac{\kappa-|\kappa|}{c}\right) .
\end{aligned}
$$

We notice the appearance of $\operatorname{sign}(\kappa)=\kappa /|\kappa|$ which is needed to distinguish between the two cases $\kappa<0$ and $\kappa>0$ in Eq. (2.13). The residuum contributions can be easily calculated analytically and with the final (innocuous) transformation $u_{1}=y_{1}+\kappa$ we obtain the following result for the two-point level correlation function (2.13),

$$
\begin{aligned}
X_{2}(r, \lambda)= & +\frac{1}{2(\pi r)^{2}}\left[\exp \left(-2 \frac{r^{2}}{\lambda^{2}}\right) \cos (2 \pi r)-1\right]+\frac{1}{(\pi \lambda)^{2}} \\
& -\left\{\frac{i}{\pi} \int_{-\infty}^{\infty} \int_{-\infty}^{\infty} d u_{1} d u_{2} \frac{\left(u_{1}-\kappa\right) u_{2}}{\left(u_{1}-\kappa\right)^{2}+\left(u_{2}-i \kappa\right)^{2}} \exp \left(-\frac{1}{2 c}\left[1-\frac{i}{u_{1}-\kappa}\right]\left(u_{1}^{2}+u_{2}^{2}\right)\right)+(\kappa \leftrightarrow-\kappa)\right\}
\end{aligned}
$$

In the limit $\lambda \rightarrow \infty$, the first two terms in Eq. (2.17) reduce to the two-point level correlation function for the GUE,

$$
X_{2}^{\mathrm{GUE}}(r)=1-\left(\frac{\sin \pi r}{\pi r}\right)^{2} .
$$

The additional terms are corrections for finite $\lambda$. We denote the double integral in Eq. (2.17) by $P(c, \kappa)$. It can be evaluated numerically for a wide range of values of $c$ and $\kappa$ without too much effort. For our present purposes, in particular for the comparison with the coset method in Sec. III, we prefer to derive some analytical results in the limit $c \ll 1$.

\section{Perturbation theory for $X_{2}(r, \lambda)$}

In the following we assume that $c \rightarrow 0$, but $\kappa=$ const. This means that the Breit-Wigner width is much larger than the level spacing, $\Gamma_{1} \gg 1$, and that the energy difference $r$ scales like $\Gamma_{1}$. Under these circumstances $P(c, \kappa)$ can be calculated perturbatively, resulting in a power series in $c$. With the polar coordinates $u_{1}=\rho \sin \varphi$ and $u_{2}=\rho \cos \varphi$ and after expanding both the exponent and the pre-exponential term in Eq. (2.17) in powers of $\rho$ we obtain the following expression for the double integral,

$$
\begin{aligned}
P(c, \kappa)= & \frac{1}{2 \pi} \int_{0}^{2 \pi} d \varphi \int_{0}^{\infty} d \rho \rho e^{i \varphi} \cos \varphi\left(1-\frac{\rho}{\kappa} \sin \varphi\right) \sum_{m=0}^{\infty}\left(\frac{\rho e^{i \varphi}}{2 \kappa i}\right)^{m} \exp \left(-\frac{i \rho^{2}}{2 c \kappa} \sum_{n=1}^{\infty}\left(\frac{\rho \sin \varphi}{\kappa}\right)^{n}\right) \times \\
& \times \exp \left(-\frac{1}{2} \frac{\kappa+i}{c \kappa} \rho^{2}\right) .
\end{aligned}
$$

The structure of the angular, i.e. the $\varphi$, integration indicates that only terms containing odd powers of $\rho$ lead to non-vanishing contributions to $P(c, \kappa)$. From Appendix A it is clear that $P(c, \kappa)$ comprises terms of order $c^{1}, c^{2}$, $c^{3}$, and so forth. For later reference, we will calculate the $c^{1}$ and $c^{2}$ contribution explicitly. To first order in $c$ the contribution of the double integral $P(c, \kappa)$ to the two-point correlator $X_{2}(r, \lambda)$ is (cf. Appendix A)

$$
P^{(1)}(c, \kappa)+P^{(1)}(c,-\kappa)=\frac{c \kappa}{2(\kappa+i)}+\frac{c \kappa}{2(\kappa-i)}=\frac{1}{\pi} \frac{\Gamma_{1}}{r^{2}+\Gamma_{1}^{2}}\left(\frac{r}{\Gamma_{1}}\right)^{2},
$$

where we have used that $\Gamma_{1}=\pi \lambda^{2}$. This result can be combined with the term $1 /\left(\pi^{2} \lambda^{2}\right)=1 /\left(\pi \Gamma_{1}\right) \equiv c$ in Eq. (2.17) to give the full first-order contribution $X_{2}^{(1)}(r, \lambda)$ to $X_{2}(r, \lambda)$, 


$$
X_{2}^{(1)}(r, \lambda)=\frac{1}{\pi} \frac{\Gamma_{1}}{r^{2}+\Gamma_{1}^{2}}
$$

The second-order contribution $X_{2}^{(2)}(r, \lambda)$ is composed of four different combinations of terms from the perturbation series (2.19), see Appendix A. Combined they can be written as

$$
\begin{aligned}
X_{2}^{(2)}(r, \lambda) & =\frac{c^{2}}{4}\left[\frac{1}{(\kappa+i)^{2}}+\frac{2 i}{(\kappa+i)^{3}}+\frac{3}{(\kappa+i)^{4}}+(\kappa \leftrightarrow-\kappa)\right] \\
& =-\frac{1}{2 \pi^{2}} \frac{\Gamma_{1}^{2}-r^{2}}{\left(r^{2}+\Gamma_{1}^{2}\right)^{2}}+\frac{c^{2}}{4}\left[\frac{2 i}{(\kappa+i)^{3}}+\frac{3}{(\kappa+i)^{4}}+(\kappa \leftrightarrow-\kappa)\right] .
\end{aligned}
$$

In the last line of the above equation we have distinguished between two contributions to $X_{2}^{(2)}(r, \lambda)$, a first piece originating from the term with the quadratic denominator, and "extra" contributions arising from the terms with higher-order denominators. The significance of this distinction will become clear in the following section, where we essentially re-derive the present results from the point of view of the coset method.

\section{COSET METHOD}

The supersymmetric "coset" method 4 , 5 has been widely employed in the last fen years or so to solve problems of random matrix theory2. In the present context, it was already used in Ref.3 for a qualitative discussion. To make contact with the well-established methods used in the literature over the years we will adopt normalization conventions in this section which differ slightly from those used in Sec. II.

\section{A. Basic definitions}

We write the full Hamiltonian $H$ in Eq. (1.1) as

$$
H=H_{0}+H_{1}
$$

thereby effectively absorbing the strength parameter $\alpha$ into the definition of $H_{1}$. The distribution $p^{(0)}(\eta)$ of the nonvanishing elements $H_{0}^{i i}$ of $H_{0}$ defines an important energy scale, the bandwidth $B$, because we have $p^{(0)}\left(H_{0}^{i i}\right) \propto B^{-1}$ for reasons of normalization. The probability distribution function $P_{N}^{(1)}\left(H_{1}\right)$ adopted here is a slightly modified version of Eq. (2.3) and reads

$$
P_{N}^{(1)}\left(H_{1}\right)=2^{N(N-1) / 2}\left(\frac{N}{2 \pi \gamma^{2}}\right)^{N^{2} / 2} \exp \left(-\frac{N}{2 \gamma^{2}} \operatorname{tr} H_{1}^{2}\right) \quad \Longrightarrow \quad\left\langle\left|H_{1}^{i j}\right|^{2}\right\rangle=\frac{\gamma^{2}}{N}
$$

where the symbol $\langle\ldots\rangle$ denotes averaging over the $H_{1}$-ensemble (i.e. the GUE). The parameter $\gamma$ introduced in this way is related to the strength parameter $\lambda$ employed in Sec. II through

$$
\lambda=\sqrt{\frac{2}{N}} \frac{\gamma}{D}
$$

It is our purpose in this section to calculate the two-point correlator $X_{2}$ (and later the number variance $\Sigma^{2}$ ) for the Hamiltonian (3.1) perturbatively in a certain suitable range of parameters. To define this range we note that $D=B / N$ is the level spacing of $H_{0}$ and $\gamma / \sqrt{N}$ the typical strength of the perturbing matrix elements. Hence we find for the (dimensionless) induced spreading or Breit-Wigner width $\Gamma_{1}$

$$
\Gamma_{1} D \propto \frac{\gamma^{2}}{N D}=\frac{\gamma^{2}}{B}
$$

Our calculation in this section is valid under the two conditions

$$
\frac{\gamma}{B}=\sqrt{\frac{\Gamma_{1} D}{B}} \rightarrow 0, \quad \Gamma_{1} \propto \frac{\gamma^{2}}{B D} \gg 1 \quad \text { (but finite) . }
$$


The first of the conditions (3.5) means that the bandwidth $B$ is infinitely larger than the Breit-Wigner width $\Gamma_{1} D$. This ensures that neither $B$ nor the level spacing $D$ are appreciably changed by the perturbation $H_{1}$. The second condition ensures that we deal with overlapping resonances, i.e. the original states of $H_{0}$ are thoroughly mixed to form the new eigenstates of the combined Hamiltonian $H$. Our perturbative calculation relies on the small parameter $1 / \Gamma_{1}$. Again, as in the previous section, the parameter $\kappa=r / \Gamma_{1}$ is held fixed so that the dimensionless energy difference $r$ scales like $\Gamma_{1}$.

With the Green function $G_{ \pm}=\left(x^{ \pm}-H\right)^{-1}$ the density of states is given by

$$
\rho(x)=\frac{1}{2 \pi}\left(\operatorname{tr} G_{+}-\operatorname{tr} G_{-}\right)
$$

and hence the averaged density-density correlation function takes the form

$$
\begin{aligned}
\rho_{2}(x+\omega / 2, x-\omega / 2) & =[\langle\rho(x+\omega / 2) \rho(x-\omega / 2)\rangle]_{0} \\
& =\frac{1}{2 \pi^{2}} \operatorname{Re}\left[\left\langle\operatorname{tr} G_{+}(x+\omega / 2)\left(\operatorname{tr} G_{-}(x-\omega / 2)-\operatorname{tr} G_{+}(x-\omega / 2)\right)\right\rangle\right]_{0} .
\end{aligned}
$$

Here, we have introduced the notation $[\ldots]_{0}$ for the $H_{0}$-average. The angular brackets $\langle\ldots\rangle$ denote, as above, averaging over $H_{1}$. In the sequel we will typically perform the $H_{1}$-average in a rather early step of the calculation while the $H_{0}$-average is performed in the final stages. We note that

$$
\rho_{2}(x+\omega / 2, x-\omega / 2)=\frac{1}{D^{2}} X_{2}(r)+\frac{1}{D^{2}} \delta(r)
$$

defines the relation of the density-density correlator to the spectral two-point function (2.9) considered earlier. Here $r=\omega / D$ is the dimensionless energy difference as in Sec. III.

\section{B. One--point function and density of states}

To illustrate our procedure and to define relevant energy scales we start with the average one-point function. With the supersymmetric generating functional

$$
\begin{aligned}
Z_{ \pm}\left(q_{ \pm}\right) & =\operatorname{detg}^{-1}\left(\left[x^{ \pm}-H\right] \otimes 1_{2}+1_{N} \otimes q_{ \pm} L_{g} / 2\right) \\
& =\exp \left(-\operatorname{trg} \ln \left(1_{N} \otimes 1_{2}+G_{ \pm} \otimes q_{ \pm} L_{g} / 2\right)\right)
\end{aligned}
$$

we can write the Green function as

$$
\operatorname{tr} G_{ \pm}(x)=\left.\frac{\partial}{\partial_{q_{ \pm}}} Z_{ \pm}\left(q_{ \pm}\right)\right|_{q=0}
$$

where $L_{g}=\operatorname{diag}(-1,1)$ is a two-dimensional (super-) matrix. With standard techniquest 1 the $H_{1}-$ average of the generating functional can be easily calculated to give

$$
\left\langle Z_{+}(J)\right\rangle=\int d[\sigma] \exp \left(-\frac{N}{2} \operatorname{trg}\left(\sigma^{2}\right)-\operatorname{trg} \ln \left(\left[x^{+}-H_{0}\right] \otimes 1_{2}+1_{N} \otimes[\gamma \sigma+J]\right)\right) .
$$

We have introduced the source matrix $J=q L_{g} / 2$, and $\sigma$ is a $2 \times 2$ supermatrix as in Eq. (2.5). In contradistinction to the previous section the further evaluation of Eq. (3.11) relies on the saddle-point approximation. With the saddle-point equation

$$
\sigma=-\frac{\gamma}{N} \sum_{j} \frac{1}{x^{+}-H_{0}^{j j}+\gamma \sigma}
$$

and with the ansatz $\gamma \sigma=\left(\Gamma_{0}+i \Gamma_{1}\right) D / 2=\Gamma D / 2$ we obtain

$$
\begin{aligned}
\Gamma & =-\frac{2 \gamma^{2}}{N D} \sum_{j} \frac{1}{x^{+}-H_{0}^{j j}+D \Gamma / 2} \\
& \approx-\frac{2 \gamma^{2}}{D} \int d \eta \frac{p^{(0)}(\eta)}{x^{+}-\eta+D \Gamma / 2} .
\end{aligned}
$$


Equation (3.13) can be solved in the limit $|\Gamma| \ll B$ and we arrive at the approximate expressions

$$
\begin{aligned}
& \Gamma_{0} \approx-\frac{2 \gamma^{2}}{D} \mathbf{P} \int d \eta \frac{p^{(0)}(\eta)}{x-\eta} \\
& \Gamma_{1} \approx 2 \pi \frac{\gamma^{2}}{D} p^{(0)}(0)=2 \pi \frac{\gamma^{2}}{B D} \quad \text { (Breit-Wigner width) } .
\end{aligned}
$$

As immediate consequences we find

$$
\begin{aligned}
\left\langle\operatorname{tr} G_{+}\right\rangle & =\operatorname{tr} \frac{1}{x^{+}-H_{0}+D \Gamma / 2}=-\frac{N \Gamma D}{2 \gamma^{2}} \\
\langle\rho(x)\rangle & =-\frac{1}{\pi} \operatorname{Im}\left\langle\operatorname{tr} G_{+}\right\rangle_{1}=\frac{N \Gamma_{1} D}{2 \pi \gamma^{2}}=N p^{(0)}(x) .
\end{aligned}
$$

In particular, the last equation means that the $H_{0}$ average of the mean level density can be trivially performed, giving $[\langle\rho(x)\rangle]_{0}=N p^{(0)}(x)$.

We see from Eq. (3.7) that we need the average of a $G_{+} G_{-}$and of a $G_{+} G_{+}$term. It is well known that in the large $N$ limit the $H_{1}$-average of the product of two Green functions with infinitesimal increments of equal sign factorizes,

$$
\left\langle\operatorname{tr} G_{+}(x+\omega / 2) \operatorname{tr} G_{+}(x-\omega / 2)\right\rangle=\left\langle\operatorname{tr} G_{+}\right\rangle\left\langle\operatorname{tr} G_{+}\right\rangle=\operatorname{tr} \frac{1}{x^{+}+\omega / 2-H_{0}+D \Gamma / 2} \operatorname{tr} \frac{1}{x^{+}-\omega / 2-H_{0}+D \Gamma / 2} .
$$

The same turns out to be true after averaging over $H_{0}$. This average is easily performed by simply integrating over the independent random entries of the diagonal matrix $H_{0}$,

$$
\begin{aligned}
{\left[\left\langle\operatorname{tr} G_{+} \operatorname{tr} G_{+}\right\rangle\right]_{0} \approx } & N(N-1) \int d \eta \frac{p^{(0)}(\eta)}{x^{+}+\omega / 2-\eta+D \Gamma / 2} \int d \eta \frac{p^{(0)}(\eta)}{x^{+}-\omega / 2-\eta+D \Gamma / 2} \\
& +N \int d \eta \frac{p^{(0)}(\eta)}{\left(x^{+}+\omega / 2-\eta+D \Gamma / 2\right)\left(x^{+}-\omega / 2-\eta+D \Gamma / 2\right)} \\
= & \frac{N(N-1)}{N^{2}}\left[\left\langle\operatorname{tr} G_{+}\right\rangle\right]_{0}\left[\left\langle\operatorname{tr} G_{+}\right\rangle\right]_{0} \approx\left[\left\langle\operatorname{tr} G_{+}\right\rangle\right]_{0}\left[\left\langle\operatorname{tr} G_{+}\right\rangle\right]_{0} .
\end{aligned}
$$

The term $\propto N$ in Eq. (3.17) is seen to vanish upon closing the contour in the lower half plane. We conclude that in order to obtain the density-density correlator (3.7) we can simply replace $\left\langle\operatorname{tr} G_{ \pm}\right\rangle=\mp i \pi / D$.

\section{Two-point function and nonlinear $\sigma$ model}

Our next goal is to calculate the remaining quantity $\left\langle\operatorname{tr} G_{+} \operatorname{tr} G_{-}\right\rangle$, which leads to the familiar nonlinear $\sigma$ model, and its $H_{0}$-average. First, we generalize the definition of the supersymmetric generating functional (3.11),

$$
\begin{aligned}
\left\langle\operatorname{tr} G_{+}(x+\omega / 2) \operatorname{tr} G_{-}(x-\omega / 2)\right\rangle & =\left.\frac{\partial}{\partial_{q_{+}}} \frac{\partial}{\partial_{q_{-}}}\left\langle Z\left(q_{+}, q_{-}\right)\right\rangle\right|_{q_{ \pm}=0}=\left.\frac{\partial}{\partial_{q_{+}}} \frac{\partial}{\partial_{q_{-}}} \int d[Q] \exp (-\mathcal{L}(Q))\right|_{q_{ \pm}=0} \\
\mathcal{L}(Q) & =\sum_{j} \operatorname{trg} \ln \left(\left[x-H_{0}^{j j}\right] 1_{4}+\frac{\omega}{2} \Lambda+\frac{D}{2} \Gamma Q+J\right) .
\end{aligned}
$$

The quantity $Q$ is a $4 \times 4$ supermatrix parameterizing the saddle-point manifold 4 国 to which the integration has been restricted by the saddle-point approximation. Furthermore, $J=\operatorname{diag}\left(q_{+} L_{g}, q_{-} L_{g}\right) / 2=q_{+} P_{+}+q_{-} P_{-}$(this defines the projectors $P_{+}$and $P_{-}$), and $\Lambda=\operatorname{diag}\left(1_{2},-1_{2}\right)$. To perform the derivatives in Eq. (3.18) it is useful to expand $\mathcal{L}$ up to second order in the source matrix $J$,

$$
\mathcal{L}(Q)=\mathcal{L}_{0}(Q)+\mathcal{L}_{1}(Q)+\mathcal{L}_{2}(Q)+\ldots
$$

The three contributions can be written as 


$$
\begin{aligned}
\mathcal{L}_{0} & =\sum_{j} \operatorname{trg} \ln \left[\left(x-H_{0}^{j j}\right) 1_{4}+\left(\omega \Lambda+\Gamma_{0} D+i \Gamma_{1} D Q\right) / 2\right] \\
\mathcal{L}_{1} & =\sum_{j} \operatorname{trg}\left[g_{j} J\right] \\
\mathcal{L}_{2} & =-\frac{1}{2} \sum_{j} \operatorname{trg}\left[g_{j} J g_{j} J\right]
\end{aligned}
$$

where we have introduced the abbreviation $g_{j}=\left[x+(\omega / 2) \Lambda-H_{0}^{j j}+\left(\Gamma_{0} D+i \Gamma_{1} D Q\right) / 2\right]^{-1}$. After performing the source term derivatives in Eq. (3.18) we arrive at a central equation of this section,

$$
\begin{aligned}
\left\langle\operatorname{tr} G_{+}(x+\omega / 2) \operatorname{tr} G_{-}(x-\omega / 2)\right\rangle & =\int d[Q]\left(S_{1}(Q)+S_{2}(Q)\right) \exp \left(-\mathcal{L}_{0}(Q)\right) \\
S_{1}(Q) & =\sum_{j, k} \operatorname{trg}\left[g_{j} P_{+}\right] \operatorname{trg}\left[g_{k} P_{-}\right] \\
S_{2}(Q) & =\sum_{j} \operatorname{trg}\left[g_{j} P_{+} g_{j} P_{-}\right] .
\end{aligned}
$$

This is the particular form of the zero-dimensional nonlinear $\sigma$ model describing the crossover between Poisson and GUE statistics.

\section{Evaluation of the nonlinear $\sigma$ model}

As already mentioned above, the $Q$-integration in Eq. (321) is restricted to the saddle-point manifold familiar from numerous previous applications of the nonlinear $\sigma$ modele. It belongs to the peculiar features of superanalysis that integrals of the type (3.21), which are derived by a supersymmetric change of variables, contain an extra "boundary" contribution (sometimes referred to as the Efetov-Wegner term). This boundary contribution is generically given by the value of the integrand at $Q=\Lambda$. In our present case we have $\mathcal{L}_{0}(\Lambda)=0, S_{2}(\Lambda)=0$, and

$$
S_{1}(\Lambda)=\sum_{j, k} \frac{1}{x-H_{0}^{j j}+D \Gamma_{0} / 2+\left(\omega+i \Gamma_{1} D\right) / 2} \frac{1}{x-H_{0}^{k k}+D \Gamma_{0} / 2-\left(\omega+i \Gamma_{1} D\right) / 2} .
$$

The $H_{0}$-average is performed as in Eq. (3.17),

$$
\begin{aligned}
S_{1}(\Lambda)= & N(N-1) \int d \eta \frac{p^{(0)}(\eta)}{x-\eta+D \Gamma_{0} / 2+\left(\omega+i \Gamma_{1} D\right) / 2} \int d \eta \frac{p^{(0)}(\eta)}{x-\eta+D \Gamma_{0} / 2-\left(\omega+i \Gamma_{1} D\right) / 2}+ \\
& +N \int d \eta \frac{p^{(0)}(\eta)}{\left(x-\eta+D \Gamma_{0} / 2+\left(\omega+i \Gamma_{1} D\right) / 2\right)\left(x-\eta+D \Gamma_{0} / 2-\left(\omega+i \Gamma_{1} D\right) / 2\right)} \\
\approx & {\left[\left\langle\operatorname{tr} G_{+}(x+\omega / 2)\right\rangle\right]_{0}\left[\left\langle\operatorname{tr} G_{-}(x-\omega / 2)\right\rangle\right]_{0}+\frac{1}{D} \int d z \frac{1}{z^{2}-\left(\omega+i \Gamma_{1} D\right)^{2} / 4} } \\
= & {\left[\left\langle\operatorname{tr} G_{+}(x+\omega / 2)\right\rangle\right]_{0}\left[\left\langle\operatorname{tr} G_{-}(x-\omega / 2)\right\rangle\right]_{0}+\frac{2 \pi}{D^{2}} \frac{1}{\Gamma_{1}-i r} . }
\end{aligned}
$$

We recall that $r=\omega / D$. The first term in Eq. (3.23 together with Eq. (3.17) forms the disconnected contribution to the two-point correlation function $X_{2}(r)$. Upon inserting the results (3.14) and (3.15) one can easily show that the sum of disconnected contributions to $X_{2}(r)$ reduces to unity (for $\Gamma_{0} \approx 0$ ). The second term in Eq. (3.23) is part of the connected contributions. In the following we calculate the remaining connected terms by treating the saddle-point integration perturbatively.

We start from Eq. (3.21). The general strategy will be to express both the exponent and the pre-exponential terms in Eq. (3.21) in terms of the independent variables of the saddle-point manifold. It turns out that we have to consider only quadratic terms in the exponent so that the integration becomes Gaussian and therefore trivial. Concerning the $H_{0}$-average it is important to note that those terms involving correlations between the matrix elements of $H_{0}$ in the exponent and in the pre-exponential terms can be neglected. In fact, it turns out that the pre-exponential terms and the exponent can be independently averaged over $H_{0}$. This amounts to a tremendous simplification of our calculation. 
A more thorough discussion of these issues as well as a number of technical steps omitted here for clarity can be found in Appendix B.

We express the deviation of $Q$ from the diagonal value $\Lambda$ in terms of the quantity $\Delta Q=\{\Lambda, Q\} / 2-1$, where $\{.,$. denotes the anticommutator. Then the $H_{0}$-average of $\mathcal{L}_{0}(Q)$ can be expressed as (see Appendix (B))

$$
\left[\mathcal{L}_{0}(Q)\right]_{0}=-i \frac{\pi}{2} \frac{r \Gamma_{1}}{\Gamma_{1}-i r} \operatorname{trg}(\Delta Q)
$$

For our present purposes, a suitable parameterization of the saddle-point manifold is given by

$$
\begin{aligned}
Q & =T^{-1} \Lambda T=\Lambda T^{2} \\
T & =\sqrt{1+R^{2}}+R \\
R & =\left[\begin{array}{ll}
0 & t \\
\bar{t} & 0
\end{array}\right]
\end{aligned}
$$

where $t$ and $\bar{t}$ are $2 \times 2$ supermatrices representing the unrestricted "free" variables of the saddle-point manifold. It follows for $\mathcal{L}_{0}$ that

$$
\left[\mathcal{L}_{0}(Q)\right]_{0}=-2 \pi i \frac{r \Gamma_{1}}{\Gamma_{1}-i r} \operatorname{trg}(\bar{t} t) .
$$

Likewise we have to express the $H_{0}$-averages of $S_{1}$ and $S_{2}$ in terms of $t$ and $\bar{t}$. Again, the detailed derivation has been deferred to Appendix B,

$$
\begin{aligned}
& {\left[S_{1}\right]_{0}=4 \frac{\pi^{2}}{D^{2}} \frac{\Gamma_{1}^{4}}{\left(\Gamma_{1}-i r\right)^{4}} \operatorname{trg}\left[t \bar{t} P_{+}\right] \operatorname{trg}\left[\bar{t} t P_{-}\right]} \\
& {\left[S_{2}\right]_{0}=-4 \frac{\pi}{D^{2}} \frac{\left(i \Gamma_{1}\right)^{2}}{\left(\Gamma_{1}-i r\right)^{3}} \operatorname{trg}\left[t P_{+} \bar{t} P_{-}\right] .}
\end{aligned}
$$

Using Eqs. 3.21), 3.26), and (3.27) the remaining Gaussian integrations can be easily performed. To this end it is useful to employ certain Wick-type contraction rules as explained in Ref. 23. Along these lines we obtain the following "diffusion" contribution from a perturbative treatment of the saddle-point integral,

$$
\left[\left\langle\operatorname{tr} G_{+} \operatorname{tr} G_{-}\right\rangle\right]_{0}^{\operatorname{diff}}=-\frac{1}{(D r)^{2}} \frac{\Gamma_{1}^{2}}{\left(\Gamma_{1}-i r\right)^{2}}+\frac{2 i \Gamma_{1}}{D^{2} r\left(\Gamma_{1}-i r\right)^{2}}=-\frac{1}{(D r)^{2}}-\frac{1}{D^{2}\left(\Gamma_{1}-i r\right)^{2}} .
$$

The individual contributions of $S_{1}$ and $S_{2}$ to this result correspond to the first and the second term on the r.h.s. of the first line, respectively.

In summary, the total $\left(H_{0}\right.$ and $\left.H_{1}\right)$ average of $\operatorname{tr} G_{+} \operatorname{tr} G_{-}$consists of three terms, the disconnected part, the boundary term (3.23), and the diffusion contribution (3.28),

$$
\left[\left\langle\operatorname{tr} G_{+} \operatorname{tr} G_{-}\right\rangle\right]_{0}=C_{\text {disc }}+C_{\text {bound }}+C_{\text {diff }}
$$

By definition, see Eqs. (3.7) and (3.8), we have

$$
X_{2}(r)=\frac{D^{2}}{2 \pi^{2}} \operatorname{Re}\left(C_{\text {bound }}+C_{\text {diff }}\right) .
$$

If we insert our results for $C_{\text {bound }}$ and $C_{\text {diff }}$ and generalize the latter to arbitrary (GUE, GOE, GSE) symmetry by multiplying it with $2 / \beta(\beta=1,2,4)$ we arrive at

$$
\begin{aligned}
X_{2}(r) & =1-\frac{1}{\beta} \frac{1}{(\pi r)^{2}}+X_{2}^{(1)}(r)+X_{2}^{(2)}(r) \\
X_{2}^{(1)}(r) & =\frac{1}{\pi} \frac{\Gamma_{1}}{\Gamma_{1}^{2}+r^{2}} \\
X_{2}^{(2)}(r) & =-\frac{1}{\pi^{2} \beta} \frac{\Gamma_{1}^{2}-r^{2}}{\left(\Gamma_{1}^{2}+r^{2}\right)^{2}} .
\end{aligned}
$$

The first term in this result is the perturbative (divergent) expression for the two-point correlation function of the Gaussian ensembles. It can be replaced by the full non-perturbative result, i.e. by Eq. (2.18) in the case of the GUE. Comparison of the first and second order contributions to $X_{2}(r), X_{2}^{(1)}(r)$ and $X_{2}^{(2)}(r)$, obtained so far with the corresponding results of the graded eigenvalue method in Eqs. (2.21) and (2.22), reveals missing terms in the present calculation. We conclude this section with a discussion of the origin of this discrepancy. 


\section{E. Fluctuating Breit-Wigner width}

Let us recall the saddle-point equation (3.13). From the first line of this equation it is clear that $\Gamma$ depends on the particular realization of $H_{0}$. But so far we have only considered one fixed (mean) value of $\Gamma$ and have neglected random fluctuations of $\Gamma$ within the ensemble. We will see below that these random fluctuations of the Breit-Wigner width lead to the missing terms in our expression for $X_{2}(\omega)$. To discuss the effect of fluctuations we choose the distribution of the (diagonal) matrix elements of $H_{0}$ to be

$$
p^{(0)}(\eta)=\frac{1}{2 W} \Theta(W-|\eta|)
$$

for definiteness. This means that the bandwidth $B$ is given by $B=2 W$. Then, according to Eq. (3.14),

$$
\begin{aligned}
& \Gamma_{0}=0 \\
& \Gamma_{1}=\frac{2 \pi \gamma^{2}}{N D^{2}} \quad(D=2 W / N) .
\end{aligned}
$$

We note that if we insert the relation (3.3) between $\gamma$ and $\lambda$ in Eq. (3.33) we obtain $\Gamma_{1}=\pi \lambda^{2}$ as claimed below Eq. (2.12). In the approximation (3.33) $\Gamma$ is given by

$$
\Gamma^{(0)}=i \Gamma_{1} .
$$

With this notation the exact saddle-point equation (3.13) reads

$$
\Gamma=-\frac{\Gamma_{1} D}{\pi} \sum_{j} \frac{1}{x-H_{0}^{j j}+D \Gamma / 2} .
$$

If we insert our lowest (zeroth) order approximation (3.34) on the r.h.s. of Eq. (3.35) we obtain the next (first) order in an iterative refinement procedure,

$$
\Gamma^{(1)}=-\frac{\Gamma_{1} D}{\pi} \sum_{j} \frac{1}{x-H_{0}^{j j}+D \Gamma^{(0)} / 2},
$$

so that the random fluctuations of $\Gamma_{c}$ are given by

$$
\delta \Gamma=\Gamma^{(1)}-\Gamma^{(0)}=-\frac{\Gamma_{1} D}{\pi} \sum_{j} \frac{1}{x-H_{0}^{j j}+i D \Gamma_{1} / 2}-i \Gamma_{1} .
$$

These fluctuations lead to additional terms in the boundary contribution because their inclusion modifies the r.h.s. of Eq. (3.22). With the definitions

$$
\begin{aligned}
G_{j} & =\frac{1}{x+\omega / 2-H_{0}^{j j}+D \Gamma^{(0)} / 2} \\
\bar{G}_{j} & =\frac{1}{x-\omega / 2-H_{0}^{j j}+D \Gamma^{(0) *} / 2}
\end{aligned}
$$

we have

$$
\begin{gathered}
\frac{1}{x+\omega / 2-H_{0}^{j j}+D\left(\Gamma^{(0)}+\delta \Gamma\right) / 2} \approx G_{j}-\frac{D}{2} \delta \Gamma G_{j}^{2} \\
\frac{1}{x-\omega / 2-H_{0}^{j j}+D\left(\Gamma^{(0) *}+\delta \Gamma^{*}\right) / 2} \approx \bar{G}_{j}-\frac{D}{2} \delta \Gamma^{*} \bar{G}_{j}^{2} .
\end{gathered}
$$

Hence we obtain instead of Eq. (3.22)

$$
\begin{aligned}
S_{1}(\Lambda) & =\sum_{j}\left(G_{j}-\frac{D}{2} \delta \Gamma G_{j}^{2}\right) \sum_{k}\left(\bar{G}_{k}-\frac{D}{2} \delta \Gamma^{*} \bar{G}_{k}^{2}\right) \\
\delta \Gamma & =-\frac{\Gamma_{1} D}{\pi} \sum_{l} G_{l}-i \Gamma_{1} \\
\delta \Gamma^{*} & =-\frac{\Gamma_{1} D}{\pi} \sum_{l} \bar{G}_{l}+i \Gamma_{1} .
\end{aligned}
$$


Averaging $S_{1}(\Lambda)$ over $H_{0}$ is tedious but in principle straightforward (see Appendix Q). The result of this calculation is that there are additional contributions to Eq. (3.23),

$$
C_{\mathrm{bound}}^{\text {extra }}=-\frac{2 \Gamma_{1}}{D^{2}\left(\Gamma_{1}-i r\right)^{3}}+\frac{3 \Gamma_{1}^{2}}{D^{2}\left(\Gamma_{1}-i r\right)^{4}},
$$

leading to the extra terms

$$
X_{2, \text { extra }}^{(2)}(\omega)=\frac{c^{2}}{4}\left[\frac{2 i}{(\kappa+i)^{3}}+\frac{3}{(\kappa+i)^{4}}+(\kappa \leftrightarrow-\kappa)\right]
$$

in the second order contribution to $X_{2}(\omega)$. These are precisely the missing terms in Eq. (3.31). We conclude that random fluctuations of the Breit-Wigner width manifest themselves in the two-point level correlation function. These extra contributions are automatically included in the exact graded eigenvalue method.

\section{NUMBER VARIANCE AND NUMERICAL SIMULATIONS}

The number variance $\Sigma^{2}(r)$ for a random matrix ensemble is defined as the variance of the number of energy levels within a dimensionless energy interval of fixed length $r$. It can be easily calculated from the spectral two-point correlation function $X_{2}(r, \lambda)$,

$$
\begin{aligned}
\Sigma^{2}(r) & =r-2 \int_{0}^{r}\left(r-r^{\prime}\right)\left(1-X_{2}\left(r^{\prime}, \lambda\right)\right) d r^{\prime} \\
& =-r(r-1)+2 \int_{0}^{r}\left(r-r^{\prime}\right) X_{2}\left(r^{\prime}, \lambda\right) d r^{\prime} .
\end{aligned}
$$

This spectral observable is particularly suitable to identify the energy scale at which the spectral statistics of the ensemble (1.1) deviates from either the GUE or Poisson behavior. For the Poisson ensemble, $\Sigma^{2}(r)$ is simply given by the straight line, $\Sigma_{\mathrm{Poi}}^{2}(r)=r$, while for the GUE the number variance is of roughly logarithmic shape. For intermediate cases, i.e. for finite non-vanishing $\alpha$ in Eq. (1.1), one expects $\Sigma^{2}(r)$ to exhibit GUE behavior for small

energy separations $r$. For larger energy intervals it should the cross over to a linear Poisson-like form. In Sec. IV A we determine, based on Eq. (4.1) and the correlator $X_{2}(r, \lambda)$ calculated in the previous sections, the energy scales relevant for this crossover. Section IVB is then devoted to the question whether our theoretical expectations are borne out by direct numerical simulations.

\section{A. Crossover Energy Scales}

The principal result of the analysis performed in Secs. II and III for the case $\Gamma_{1} \gg 1$ is that the two-point level correlator $X_{2}(r, \lambda)$ for the random matrix model (1.1) can be written as

$$
\begin{aligned}
X_{2}(r, \lambda) & =X_{2}^{\mathrm{GUE}}(r)+X_{2}^{\mathrm{NA}}(r, \lambda)+X_{2}^{(1)}(r, \lambda)+X_{2}^{(2)}(r, \lambda) \\
X_{2}^{\mathrm{GUE}}(r) & =1-\frac{\sin ^{2}(\pi r)}{(\pi r)^{2}}=1-\frac{1}{2 \pi^{2} r^{2}}(1-\cos (2 \pi r)) \\
X_{2}^{\mathrm{NA}}(r, \lambda) & =\frac{1}{2 \pi^{2} r^{2}}\left[\exp \left(-2 \pi \frac{r^{2}}{\Gamma_{1}}\right)-1\right] \cos (2 \pi r) \\
X_{2}^{(1)}(r, \lambda) & =\frac{1}{\pi} \frac{\Gamma_{1}}{\Gamma_{1}^{2}+r^{2}} \\
X_{2}^{(2)}(r, \lambda) & =-\frac{1}{2 \pi^{2}} \frac{\Gamma_{1}^{2}-r^{2}}{\left(\Gamma_{1}^{2}+r^{2}\right)^{2}}+\frac{1}{2 \pi^{2}}\left(\frac{-2 \Gamma_{1}^{2}\left(\Gamma_{1}^{2}-3 r^{2}\right)}{\left(\Gamma_{1}^{2}+r^{2}\right)^{3}}+\frac{3 \Gamma_{1}^{2}\left(\Gamma_{1}^{4}-6 \Gamma_{1}^{2} r^{2}+r^{4}\right)}{\left(\Gamma_{1}^{2}+r^{2}\right)^{4}}\right)
\end{aligned}
$$


with $\Gamma_{1}=\pi \lambda^{2}=\pi(\alpha / D)^{2}$. The first contribution $X_{2}^{\mathrm{GUE}}(r)$ is just the two-point level correlator for the GUE case. The contribution $X_{2}^{\mathrm{NA}}(r, \lambda)$ describes nonanalytic corrections for $\Gamma_{1}^{-1} \ll 1$ (with $r$ of order $\Gamma_{1}$ ) that cannot be obtained by perturbation theory. They are found in the framework of the graded eigenvalue method or a more sophisticated nonperturbative evaluation of the coset method. This contribution tends to eliminate the oscillating part of $X_{2}^{\mathrm{GUE}}(r)$ for $r \gg \sqrt{\Gamma_{1}}$. The terms $X_{2}^{(1)}(r, \lambda)$ and $X_{2}^{(2)}(r, \lambda)$ are just the first or second order corrections in $\Gamma_{1}^{-1}$. In (4.2), we have focused on the unitary case $(\beta=2)$ for definiteness. Our qualitative conclusions, however, apply likewise to the orthogonal and the symplectic symmetry class $(\beta=1,4)$. The nonanalytic correction can be neglected when performing the $r^{\prime}$-integral in (4.1) to determine the number variance. As a consequence of the form of Eq. (4.2) we obtain

$$
\Sigma^{2}(r, \lambda)=\Sigma_{\mathrm{GUE}}^{2}(r)+\Sigma_{(1)}^{2}(r, \lambda)+\Sigma_{(2)}^{2}(r, \lambda) .
$$

A short calculation using Eqs. (4.1) and (4.2) reveals that

$$
\Delta \Sigma^{2}(r, \lambda)=\Sigma_{(1)}^{2}(r, \lambda)+\Sigma_{(2)}^{2}(r, \lambda)=\frac{2 r}{\pi} \arctan \left(\frac{r}{\Gamma_{1}}\right)-\left(\frac{\Gamma_{1}}{\pi}+\frac{1}{2 \pi^{2}}\right) \ln \left(1+\left(\frac{r}{\Gamma_{1}}\right)^{2}\right)+\frac{1}{2 \pi^{2}} \frac{r^{2} \Gamma_{1}^{2}-r^{4}}{\left(r^{2}+\Gamma_{1}^{2}\right)^{2}} .
$$

It is consistent with our assumption $\Gamma_{1} \gg 1$ to neglect the second term $1 / 2 \pi^{2}$ in the prefactor of the logarithmic contribution and the last term in Eq. (4.4). To identify the energy scales of interest we first consider the limit $r \ll \Gamma_{1}$. Then

$$
\Delta \Sigma^{2}(r, \lambda) \approx \frac{2 r^{2}}{\pi \Gamma_{1}}-\frac{\Gamma_{1}}{\pi}\left(\frac{r}{\Gamma_{1}}\right)^{2}=\frac{r^{2}}{\pi \Gamma_{1}} \quad\left(r \ll \Gamma_{1}\right) .
$$

Obviously, the scale set for deviations from the GUE behavior is $\sqrt{\Gamma_{1}}$ in agreement with previous findings 20 .

Let us now ask a different question, namely, what is the limit of Eq. (4.4) for $r \gg \Gamma_{1}$ and how is it approached? We expand the arcustangent in Eq. (4.4) for large argument and arrive at

$$
\Delta \Sigma^{2}(r, \lambda) \approx r-\frac{2}{\pi} \Gamma_{1}-\frac{\Gamma_{1}}{\pi} \ln \left(1+\left(\frac{r}{\Gamma_{1}}\right)^{2}\right) .
$$

Apart from a constant and logarithmic corrections in $r$ (in this context the GUE result $\Sigma_{\mathrm{GUE}}^{2}(r)$ to be added to Eq. (4.6) is also nothing but a logarithmic correction) we recover the Poisson limit $\Sigma_{\text {Poi }}^{2}(r)=r$. The energy scale governing the asymptotic approach to the Poisson limit is given by $\Gamma_{1}$. We can see this by dividing Eq. (4.4) by $r$. Within our usual approximations we find

$$
\frac{1}{r} \Delta \Sigma^{2}(r, \lambda)=\frac{2}{\pi} \arctan \left(\frac{r}{\Gamma_{1}}\right)-\frac{\Gamma_{1}}{\pi r} \ln \left(1+\left(\frac{r}{\Gamma_{1}}\right)^{2}\right) .
$$

This function approaches 1 for $r \gg \Gamma_{1}$ and depends only on the ratio $r / \Gamma_{1}$. Hence the relevant energy scale is $\Gamma_{1}$ as claimed.

It is quite instructive to compare our results with the case of a disordered metal in the diffusive limit. The twopoint level correlator for this case was calculated by Al'tshuler and Shklovskii24 in the framework of diagrammatic perturbation theory,

$$
X_{2}\left(\frac{\omega}{D}\right)=1+\frac{D^{2}}{\beta \pi^{2}} \operatorname{Re} \sum_{\mathbf{q}} \frac{1}{\left(-i \omega+\mathcal{D}_{0} \mathbf{q}^{2}\right)^{2}}
$$

where $\beta=1,2,4$ labels the symmetry class and $\mathcal{D}_{0}$ is the diffusion constant (note that $D$ is the mean level spacing). This expression is valid for $\omega \gg D$. The sum extends over the diffuson modes characterized by wave vectors $\mathbf{q}$. The mode $\mathbf{q}=0$ corresponds to random matrix theory while the modes $\mathbf{q} \neq 0$ give the deviations from random matrix behavior. The latter can be described by a function $f(x)$ in the following way

$$
X_{2}\left(\frac{\omega}{D}\right)=1-\frac{D^{2}}{\beta \pi^{2} \omega^{2}}+\frac{D^{2}}{E_{c}^{2}} f\left(\frac{\omega^{2}}{E_{c}^{2}}\right), \quad f(x)=a_{0}+a_{2} x^{2}+\ldots .
$$

Here $E_{c} \sim \mathcal{D}_{0} / L^{2}$ is the diffusive Thouless energy for a sample of size $L$. The coefficients $a_{0}, a_{2}, \ldots$ and therefore the function $f(x)$ depend only on the space dimension $d$ and on the particular geometry of the sample but not on any 
other physical parameter. These coefficients are given by discrete sums over "integer" wave vectors which converge nicely for $d \leq 3$. The correction of the nonzero modes to the number variance is immediately estimated as

$$
\Delta \Sigma^{2}(r) \sim \frac{r^{2}}{\left(E_{c} / D\right)^{2}}+\ldots
$$

with $r=\omega / D$. This corrections is of order 1 just for $r \sim E_{c} / D$ and not $\sqrt{E_{c} / D}$. Here the crossover is indeed governed by only one parameter and there is no transition regime like the interval between $\sqrt{\Gamma_{1}}$ and $\Gamma_{1}$ as found above.

\section{B. Comparison with Numerical Spectra}

For a verification of our analytical calculations we numerically generate a matrix ensemble of the type (1.1). The distribution function $p^{(0)}(\eta)$ of the independent elements of the diagonal matrix $H_{0}$ is chosen to be a semicircle with radius $R_{0}=30$ (in arbitrary energy units). The matrix $H_{0}$ is perturbed by a GUE ensemble $H_{1}$ with $\left\langle\left|H_{1}^{i j}\right|^{2}\right\rangle=\alpha^{2} / 2$ as in Eq. (1.1). We consider matrices of dimension $N=500$ and for each set of values $\alpha, R_{0}$ we take 500 realizations into account. To ensure that $\Gamma_{1}>D$ in our simulation we take the parameter $\alpha$ from the interval [0.1,3.0]. Larger values of $\alpha$ are no longer useful because it is difficult to distinguish the ensuing spectral statistics from pure GUE statistics.

The spectra obtained by diagonalization of $H=H_{0}+H_{1}$ are unfolded numerically. Then the number variance $\Sigma_{\mathrm{sim}}^{2}(r)$ is calculated for a number of different values of $\alpha$. Given this set of numerical functions we fit (by hand) the theoretical formula given by Eqs. (4.3) and (4.4) to the data. This allows to determine the unfolded crossover parameter $\lambda$ (via the relation $\Gamma_{1}=\pi \lambda^{2}$ ). Furthermore, we also extract the "critical" energy scale $r_{c}$ at which $\Sigma_{\text {sim }}^{2}(r)$ deviates from GUE behavior from the data. We define $r_{c}$ by the condition

$$
\frac{\Sigma_{\mathrm{sim}}^{2}\left(r_{c}\right)-\Sigma_{\mathrm{GUE}}^{2}\left(r_{c}\right)}{\Sigma_{\mathrm{GUE}}^{2}\left(r_{c}\right)}=0.2
$$

which means that at the point $r_{c}$ the actual (simulated or fitted) number variance exceeds $\Sigma_{\text {GUE }}^{2}\left(r_{c}\right)$ by 20 percent. Of course, this condition is somewhat arbitrary. However, in our opinion it correctly reflects what we usually mean by a "significant deviation from GUE behavior". By means of the procedure just described a given value of $\alpha$ is associated with particular values of $\lambda$ and $r_{c}$. The following figures illustrate the procedure and our results.

In Fig. 2 we show the numerically calculated number variance $\Sigma_{\operatorname{sim}}^{2}(r)$ and the corresponding fit for the two values $\alpha=0.5$ and $\alpha=1.2$. In the latter case, there is very satisfactory agreement up to very large energy separations of $r=140$. In the former case, we find agreement of this quality up to $r=30$. Beyond this value, we find some disagreement which is likely to be due to the limits of our approximation which relies on large $\lambda$ values (i.e. $\alpha \gg D$ ). Fig. 3 shows the fitted crossover parameter $\lambda$ and the theoretical value $\lambda_{\text {th }}=\alpha / D$. For small values $\alpha \lesssim 0.5$ both curves agree well. The deviations for $\alpha \gtrsim 0.5$ are due to the fact that the Breit-Wigner width $\Gamma_{1} D$ already approaches the effective bandwidth of the unperturbed spectrum. In fact the radius $R_{0}$ of the semicircle density equals $\Gamma_{1} D$ for $\alpha_{c} \approx 0.95$. In this regime both theoretical approaches are strictly speaking not valid due to the condition (3.5). However, it seems that the theoretical expression for the number variance remains even here correct, if the parameter $\lambda$ is correspondingly rescaled ("fitted"). Fig. 团 shows the dependence of the critical energy separation $r_{c}$ on $\alpha$. Two observations are important: First, the dependence is by no means linear. Second, the data seems to indicate a crossover between two power-laws (see inset for a logarithmic plot), which occurs around $\alpha \approx 0.6-0.7$. The approximate power-law exponents in the two regions are roughly given by 1.2 and 2.2 , respectively. Fig. 5 , on the other hand, demonstrates that the dependence of $r_{c}$ on $\lambda$ is almost perfectly linear as expected on theoretical grounds and does not exhibit any crossover behavior. The dashed line in Fig. 5 represents the theoretical energy scale $\sqrt{\Gamma_{1}}=\sqrt{\pi} \lambda$. We see that the 20 percent criterion gives roughly $r_{c} \approx 0.7 \sqrt{\Gamma_{1}}$.

The difference between Figs. 1 and 5 is due to the deviations shown in Fig. 3 for $\alpha \gtrsim 0.5$. This effect is related to the finite bandwidth being comparable to the Breit-Wigner width and produces the crossover in Fig. 4 . In this context, we mention another effect which can play a role in such numerical simulations. The mean level spacing of the matrix ensemble (1.1) in general depends on $\alpha$. For large enough values of this parameter the perturbation $\alpha H_{1}$ in Eq. (1.1) will start to increase the overall bandwidth of the spectrum and hence the mean level spacing. Therefore the dimensionless critical energy $r_{c}(\alpha)=\omega_{c}(\alpha) / D(\alpha)$ in general receives contributions from both the numerator and denominator.

In Fig. 5, we do not observe any crossover behavior showing that $r_{c}$ is a linear function of $\lambda$. This confirms our previous analytical result that the relevant energy scale for deviations from the GUE is given by $\sqrt{\Gamma_{1}} \propto \lambda$. 


\section{SUMMARY AND CONCLUSIONS}

The main results of the present paper are the exact expression for $X_{2}(r)$ in terms of a handy double integral in Eq. (2.17), the perturbative formula (3.31) for $X_{2}(r)$ including the extra terms (3.42), and the perturbative expressions for $\Sigma^{2}(r)$ in Eqs. (4.3) and (4.4). Our calculations show that the spectral properties of the Hamiltonian $H=H_{0}+\alpha H_{1}$ are governed by two energy scales, the Breit-Wigner width $\Gamma_{1}$ and its square root $\sqrt{\Gamma_{1}}$. While the former sets the scale for deviations from the Poisson limit, the latter determines the scale at which the difference to GUE statistics becomes noticeable.

To derive these results we employed two supersymmetric approaches, the graded eigenvalue method and the coset method. For the technically interested expert, the problem considered in this paper offers the possibility to compare both methods in some detail. As a new physical insight from this comparison, we could show that random fluctuations of the Breit-Wigner width lead to certain extra contributions to the spectral correlation functions. These contributions are automatically included in the (exact) graded eigenvalue method. They are also included in the coset method but here the discrete saddle point equation requires a very careful treatment when applying the additional average with respect to the diagonal elements because the Breit-Wigner width exhibits small random fluctuations when those diagonal elements are varied.

We briefly discuss the relevance and implications of this paper for previously published work. In Ref. 21 a diagrammatic analysis of the two-level correlation function $X_{2}(r)$ was presented. There, a central aim was to provide a physically more transparent derivation of the results published in Ref. 20. Comparing our formula (2.22) with the expression (15) in Ref. 21 it seems that the results of Ref. 21 are qualitatively, but not quantitatively, correct. This discrepancy, which appears for the second order terms, is due to the contributions arising from the statistical fluctuations of the Breit-Wigner width (see Sec. IIIE and Appendix Ø) which are completely neglected in Ref. 21. To understand this in detail, we have also to take into account that Altland et al. considered a model where the diagonal matrix elements of the perturbation $\alpha H_{1}$ are zero. We have therefore modified their diagrammatic calculation for our case where $H_{1}$ is a GUE-matrix with non-vanishing diagonal matrix elements. The result is in perfect agreement with Eq. (3.31) obtained by the $\sigma$ model perturbation theory. Adding the extra terms (3.41) from the fluctuating Breit-Wigner width, we again recover our result (2.22) from the graded eigenvalue method.

However, we expect that the model of Altland et al. (with $H_{1}^{j j}=0$ ) should be equivalent to our case since, in the limit $D \Gamma_{1} / B \rightarrow 0$, the diagonal elements can be absorbed by an infinitesimal rescaling of $H_{0}$. Inspecting Eq. (15) of Ref. 21, we see that here the diffuson perturbation theory already contains the first extra contribution (C12) but not the second extra term ( $\mathrm{C25})$. The reason is that for the model with $H_{1}^{j j}=0$ the fluctuations of $\Gamma$ appear in a different way. Indeed, the Dyson (or saddle point) equation is now slightly modified

$$
\Gamma(k)=-\frac{\Gamma_{1} D}{\pi} \sum_{j(\neq k)} \frac{1}{x-H_{0}^{j j}+D \Gamma(j) / 2} .
$$

Note that $\Gamma(k)$ depends on the site index and that the sum extends only over the $j$ being different from $k$. In the approximation (3.13) this gives no difference while the treatment of Appendix $\mathbf{Q}$ is modified. It turns out that now the first term ( $\mathrm{C} 12)$ vanishes while the second contribution $(\mathrm{C} 25)$ still persists. Together with the contributions from the diffuson diagrams [Eq. (15) of Ref. 21], we obtain again perfect agreement with Eq. (2.22).

Apparently, for both models with and without diagonal matrix elements in $H_{1}$ there are second order contributions arising from both the diagrammatic diffuson terms and the fluctuations of the Breit-Wigner width. The sum of these contributions is in both cases the same as it should be. However, the way they appear as diffuson terms or $\Gamma$-fluctuations is different.

In Ref. 13 numerical and analytical evidence was reported for a crossover from a linear to a quadratic dependence of the critical energy $r_{c}$ on the strength of the-perturbing (GOE) matrix elements. From our calculations we cannot confirm such a behavior. Both our previous 20 and present analysis indicate the the critical energy $r_{c}$ is linear in $\lambda$, irrespective of the range, $\lambda>1$ or $\lambda<1$ (see Ref. 20), considered. Fig. 4 gives the (erroneous) impression of a crossover because the strength parameter $\alpha$ is not properly measured on the unfolded scale. The fact that the mean level spacing of the Hamiltonian (1.1) depends on $\alpha$ necessitates an $\alpha$-dependent unfolding. This leads to the linear behavior in Fig. 5.

Finally, we would like to re-emphasize that the ensemble (1.1) simulates a very general situation: A diagonalized quantum system with Poisson statistics is perturbed by a non-diagonal random operator. Situations where this model system is appropriate should abound, and in these cases the spectral behavior predicted here, in particular the energy scales $\sqrt{\Gamma_{1}}$ and $\Gamma_{1}$ should be readily identifiable. 


\section{ACKNOWLEDGMENT}

It is a pleasure to thank Shinji Iida for his interest in our work and very valuable and helpful discussions and remarks.

\section{APPENDIX A: PERTURBATIVE EVALUATION OF EQ. (2.19)}

In this Appendix we compute the terms of order $c^{1}$ and $c^{2}$ in the perturbative expansions of the integral $P(c, \kappa)$ defined in Eq. (2.19). For this we keep in both sums in Eq. (2.19) the terms up to order $\rho^{2}$,

$$
P(c, \kappa)=\frac{1}{2 \pi} \int_{0}^{2 \pi} d \varphi \int_{0}^{\infty} d \rho \rho e^{i \varphi} \cos \varphi\left(1-\frac{\rho}{\kappa} \sin \varphi\right)\left(1+\frac{\rho e^{i \varphi}}{2 \kappa i}+\left(\frac{\rho e^{i \varphi}}{2 \kappa i}\right)^{2}+\cdots\right) e^{-L_{0}-L_{1}-L_{2}-\cdots}
$$

with

$$
L_{0}=\frac{\rho^{2}}{2 \Pi} \quad, \quad \Pi=\frac{c k}{k+i} \quad, \quad L_{n}=\frac{i \rho^{2}}{2 c \kappa}\left(\frac{\rho \sin \varphi}{\kappa}\right)^{n} \quad \text { if } n \geq 1
$$

Here we have introduced the notion $\Pi$ for the "propagator" of the perturbation theory. To keep a compact notation we introduce the abbreviation

$$
\langle f(\rho, \varphi)\rangle_{(\rho, \varphi)}=\frac{1}{2 \pi} \int_{0}^{2 \pi} d \varphi \int_{0}^{\infty} d \rho \rho e^{-\rho^{2} /(2 \Pi)} f(\rho, \varphi),
$$

where $f(\rho, \varphi)$ stands for an arbitrary function of $\rho$ and $\varphi$. Expanding the exponential in (A1) with respect to $L_{1}$ and $L_{2}$, we obtain

$$
P(c, \kappa)=\left\langle e^{i \varphi} \cos \varphi\left(1+\frac{\rho e^{-i \varphi}}{2 \kappa i}+\left(\frac{\rho}{2 \kappa i}\right)^{2}+\cdots\right)\left(1-L_{1}-L_{2}+\frac{1}{2} L_{1}^{2}+\cdots\right)\right\rangle_{(\rho, \varphi)} .
$$

Obviously, in (A4) the terms with odd power in $\rho$ vanish while the terms with even power can be evaluated using $\left\langle\rho^{2 m}\right\rangle_{(\rho, \varphi)}=\Pi^{m+1} 2^{m} m$ !. The $c^{1}$-term is therefore given by

$$
P^{(1)}(c, \kappa)=\left\langle e^{i \varphi} \cos \varphi\right\rangle_{(\rho, \varphi)}=\frac{1}{2} \Pi
$$

in agreement with Eq. 2.20). There are four different second order terms,

$$
\begin{aligned}
& P_{a}^{(2)}(c, \kappa)=\left\langle e^{i \varphi} \cos \varphi\left(\frac{\rho}{2 \kappa i}\right)^{2}\right\rangle_{(\rho, \varphi)}=-\frac{1}{4 \kappa^{2}}\left\langle\rho^{2} \cos ^{2} \varphi\right\rangle_{(\rho, \varphi)}=-\frac{\Pi^{2}}{4 \kappa^{2}}, \\
& P_{b}^{(2)}(c, \kappa)=-\left\langle e^{i \varphi} \cos \varphi \frac{\rho e^{-i \varphi}}{2 \kappa i} L_{1}\right\rangle_{(\rho, \varphi)}=-\frac{1}{4 c \kappa^{3}}\left\langle\rho^{4} \cos \varphi \sin \varphi\right\rangle_{(\rho, \varphi)}=0, \\
& P_{c}^{(2)}(c, \kappa)=-\left\langle e^{i \varphi} \cos \varphi L_{2}\right\rangle=-\frac{i}{2 c \kappa^{3}}\left\langle\rho^{4} \cos ^{2} \varphi \sin ^{2} \varphi\right\rangle=-\frac{i}{2 c \kappa^{3}} 2 ! \cdot 2^{2} \Pi^{3} \frac{1}{8}=-\frac{i}{2 c \kappa^{3}} \Pi^{3}, \\
& P_{d}^{(2)}(c, \kappa)=\frac{1}{2}\left\langle e^{i \varphi} \cos \varphi L_{1}^{2}\right\rangle=-\frac{1}{8 c^{2} \kappa^{4}}\left\langle\rho^{6} \cos ^{2} \varphi \sin ^{2} \varphi\right\rangle=-\frac{1}{8 c^{2} \kappa^{4}} 3 ! \cdot 2^{3} \Pi^{4} \frac{1}{8}=-\frac{3}{4 c^{2} \kappa^{4}} \Pi^{4},
\end{aligned}
$$

which result in

$$
P^{(2)}(c, \kappa)=\sum_{\alpha=a, b, c, d} P_{\alpha}^{(2)}(c, \kappa)=-\frac{\Pi^{2}}{4 \kappa^{2}}\left(1+2 i\left(\frac{\Pi}{c \kappa}\right)+3\left(\frac{\Pi}{c \kappa}\right)^{2}\right)
$$

Inserting $\Pi=c \kappa /(\kappa+i)$, we obtain Eq. (2.22). 


\section{APPENDIX B: PERTUBATION THEORY IN THE FRAME WORK OF THE COSET METHOD}

The precise $\sigma$ model action $\mathcal{L}_{0}$ and the preexponential terms in (3.21) still depend on the particular realisation of the diagonal matrix elements $H_{0}^{j j}$. The aim of this appendix is to show how one can perform in a suitable way the average with respect to these matrix elements and how the resulting $Q$-integral can be evaluated perturbatively for $Q$ being close the origin $\Lambda$ in the coset space. In order to expand $\mathcal{L}_{0}$ with respect to $\Delta Q=\{\Lambda, Q\} / 2-1=2 R^{2}$, we consider the quantity

$$
I=\operatorname{trg} \ln (a+b \Lambda+c Q)
$$

with some spmplex parameters $a, b, c$ and $\Lambda, Q$ as defined in section III q. The quantity $I$ only depends on the radial parameter $\theta$ of $Q=T^{-1} \Lambda T$, i.e. we may choose $T=\exp \left(\theta \sigma_{1}\right)$, where $\sigma_{1}=\left(\begin{array}{ll}0 & 1 \\ 1 & 0\end{array}\right)$ and $\theta$ is $2 \times 2$ supermatrix with two independent radial parameters 1 (for the unitary symmetry class). Then we have $\sigma_{1} \Lambda \sigma_{1}=-\Lambda$ and $\sigma_{1} Q \sigma_{1}=-Q$. Inserting $1=\sigma_{1}^{2}$ and permuting the matrix products in the argument of the logarithm in (B1), we thus obtain:

$$
\begin{aligned}
I & =\operatorname{trg} \ln (a-b \Lambda-c Q)=\frac{1}{2} \operatorname{trg} \ln ((a+b \Lambda+c Q)(a-b \Lambda-c Q))= \\
& =\frac{1}{2} \operatorname{trg} \ln \left(\left[a^{2}-(b+c)^{2}\right]-b c[Q \Lambda+\Lambda Q-2]\right)=\frac{1}{2} \operatorname{trg} \ln \left(\left[a^{2}-(b+c)^{2}\right]-2 b c \Delta Q\right) \\
& =\frac{1}{2} \operatorname{trg} \ln \left(1-\frac{2 b c}{a^{2}-(b+c)^{2}} \Delta Q\right) .
\end{aligned}
$$

Using this identy, we can rewrite $\mathcal{L}_{0}$ as

$$
\mathcal{L}_{0}=\frac{1}{2} \sum_{j} \operatorname{trg} \ln \left(1-\frac{i \omega \Gamma_{1} D / 2}{\left(x-H_{0}^{j j}+\Gamma_{0} D / 2\right)^{2}+\left(\Gamma_{1} D / 2-i \omega / 2\right)^{2}} \Delta Q\right) .
$$

The logarithm can be exanded in powers of $\Delta Q$ giving $\mathcal{L}_{0}=\mathcal{L}_{0}^{(2)}+\mathcal{L}_{0}^{(4)}+\cdots$ where $\mathcal{L}_{0}^{(2)}$ is the gaussian part of the action. Furthermore we note that in the limit $\Gamma_{1} \gg 1$ the discrete $j$-sum consists of Lorentzians with well overlapping resonances which allows to replace in lowest order of $\Gamma_{1}^{-1}$ the gaussian part $\mathcal{L}_{0}^{(2)}$ by its average over the diagonal elements $H_{0}^{j j}$. Therefore we obtain, in the limit $B / \Gamma_{1} \rightarrow \infty$,

$$
\mathcal{L}_{0}^{(2)}(Q) \approx\left[\mathcal{L}_{0}^{(2)}(Q)\right]_{0}=-i \frac{\pi}{D} \frac{\omega \Gamma_{1} / 2}{\Gamma_{1} D-i \omega} \operatorname{trg}(\Delta Q)=-i \frac{\pi}{2} \frac{r \Gamma_{1}}{\Gamma_{1}-i r} \operatorname{trg}(\Delta Q)
$$

where $r=\omega / D$ and $B$ is the bandwidth over which the diagonal matrix elements $H_{0}^{j j}$ are distributed. Omitting the details, we mention that one can show in a more refined way that the statistical fluctuations indeed yield corrections of lower order in $\Gamma_{1}^{-1}$. For this, one has to analyze the average of the quantity $\exp \left(-\mathcal{L}_{0}^{(2)}(Q)\right)$ more carefully.

The spirit of the pertubative calculation consists of expanding the the preexponential terms [and also of the nongaussian terms $\exp \left(-\mathcal{L}_{0}^{(4)}-\cdots\right)$ ] with respect to the variables $R$ (or $\left.t\right)$ [see (3.25)]. Then one can systematically evaluate the gaussian integrals using Wick's theorem. Each pair of $t$ and $\bar{t}$ yields here a power $\Pi_{D}^{-1}$ of the propagator which is defined by the prefactor of the gaussian action

$$
\mathcal{L}_{0}^{(2)}(Q) \approx-\pi i \frac{r \Gamma_{1}}{\Gamma_{1}-i r} \operatorname{trg}\left(R^{2}\right)=-2 \pi i \frac{r \Gamma_{1}}{\Gamma_{1}-i r} \operatorname{trg}(\bar{t} t)=\Pi_{D} \operatorname{trg}(\bar{t} t)
$$

The perturbation theory is justified for $\left|\Pi_{D}\right| \gg 1$ which is certainly correct if $\Gamma_{1} \gg 1$ and if the ratio $\kappa=r / \Gamma_{1}$ is finite and non 0 . As in the pure GUE case, this simple perturbation theory does not allow to access the very small frequencies $\omega \sim D$. We emphasize that this is different for the pertubative evaluation of the integral $(2.19)$ which was obtained in the frame work of the precise graded eigenvalue method in section II (see appendix A). Here the pertubative condition only consists of $\Gamma_{1} \gg 1$ and the behavior for small $\omega$ is precisely described by the residuum contributions in (2.16) which even contain the nonanalytical corrections.

We have to expand $g_{j}$ in the preexponential factors in (3.21) with respect to the small quantity

$$
\delta Q=Q-\Lambda=2 \Lambda R \sqrt{1+R^{2}}+2 \Lambda R^{2}=2 \Lambda R+2 \Lambda R^{2}+\cdots
$$

which gives 


$$
g_{j}=g_{j}^{(0)}+\left(i \frac{\Gamma_{1} D}{2}\right) g_{j}^{(0)} \delta Q g_{j}^{(0)}+\left(i \frac{\Gamma_{1} D}{2}\right)^{2} g_{j}^{(0)} \delta Q g_{j}^{(0)} \delta Q g_{j}^{(0)}+\cdots
$$

where

$$
\begin{aligned}
& g_{j}^{(0)}=\left[x-H_{0}^{j j}+\Gamma_{0} D / 2+i \Lambda\left(\Gamma_{1} D-i \omega\right) / 2\right]^{-1}, \\
& \bar{g}_{j}^{(0)}=\left[x-H_{0}^{j j}+\Gamma_{0} D / 2-i \Lambda\left(\Gamma_{1} D-i \omega\right) / 2\right]^{-1} .
\end{aligned}
$$

To evaluate the term $S_{1}(Q)$ in (3.21), we consider

$$
\begin{aligned}
\sum_{j} \operatorname{trg}\left(g_{j} P_{ \pm}\right)= & \sum_{j} \operatorname{trg}\left(g_{j}^{(0)} P_{ \pm}\right)+\left(i \frac{\Gamma_{1} D}{2}\right) \sum_{j} \operatorname{trg}\left[\left(g_{j}^{(0)}\right)^{2}\left(2 \Lambda R+2 \Lambda R^{2}\right) P_{ \pm}\right] \\
& +\left(i \frac{\Gamma_{1} D}{2}\right)^{2} \sum_{j} \operatorname{trg}\left[\left(g_{j}^{(0)}\right)^{2} \bar{g}_{j}^{(0)}(2 \Lambda R 2 \Lambda R) P_{ \pm}\right]+\cdots \\
= & : A_{0}^{( \pm)}+A_{1}^{( \pm)}+A_{2}^{( \pm)}+
\end{aligned}
$$

where we have used that $g_{j}^{(0)} R=R \bar{g}_{j}^{(0)}$ since $\Lambda R \Lambda=-R$. The term $S_{1}(Q)$ is now given by

$$
S_{1}(Q)=\left(A_{0}^{(+)}+A_{1}^{(+)}+A_{2}^{(+)}\right)\left(A_{0}^{(-)}+A_{1}^{(-)}+A_{2}^{(-)}\right)
$$

The main contribution arises from the product $A_{0}^{(+)} A_{0}^{(-)}$which is effectively of order $\Pi_{D}^{-0}$ and also contains terms of higher orders $\Pi_{D}^{-1}, \Pi_{D}^{-2}, \ldots$. This contribution just corresponds to the boundary terms (3.22) which are extensively discussed in subsection IIIE and appendix C. Here we concentrate on the higher order terms arising from $A_{1}$ and $A_{2}$ which are both of second order in $R$ (the linear $R$ term in $A_{1}$ vanishes under the graded trace). They will give contributions of order $\Pi_{D}^{-2}$ which is the highest order in which we are interested. (This corresponds to order $c^{2}$ in appendix A.) Therefore we can here replace the correlated $H_{0}$-averages like $\left[A_{2} A_{2} \exp \left(-\mathcal{L}_{0}^{(2)}\right)\right]_{0}$ by $\left[A_{2}\right]_{0}\left[A_{2}\right]_{0} \exp \left(-\left[\mathcal{L}_{0}^{(2)}\right]_{0}\right)$ since the correlations arising from the diagonal terms or the exponential are already of order $\Pi_{D}^{-3}$. The crossed terms like $\left[A_{0} A_{2}\right]_{0}$ are in principle of order $\Pi_{D}^{-1}$ but they vanish upon the gaussian contraction rules (for details see Ref. 23). Actually, they are of the type $\operatorname{trg}\left(t \bar{t} P_{+}\right) \rightarrow \Pi_{D}^{-1} \operatorname{trg}(1) \operatorname{trg}\left(P_{+}\right)=0$. The product of these terms with first term arising form the nongaussian part of the action, i.e. $\left[A_{0} A_{2}\right]_{0} \mathcal{L}_{0}^{(4)}$, is of order $\Pi_{D}^{-2}$ and of the type $\operatorname{trg}\left(t \bar{t} P_{+}\right) \operatorname{trg}(t \bar{t} t \bar{t})$ which also vanishes. The term $\left[A_{1}\right]_{0}\left[A_{1}\right]_{0}$ vanishs upon the $H_{0}$-average while $\left[A_{2}\right]_{0}\left[A_{2}\right]_{0}$ gives the only nonvanishing contribution. The $H_{0}$-average can be performed using the integrals (C6).C8) evaluated in appendix Q:

$$
A_{2}^{( \pm)} \rightarrow\left[A_{2}^{( \pm)}\right]_{0}=\left(i \frac{\Gamma_{1} D}{2}\right)^{2} \frac{(-2 \pi i)}{D} \frac{1}{\left(\Gamma_{1} D-i \omega\right)^{2}} \operatorname{trg}\left(-4 \Lambda R^{2} P_{ \pm}\right)=-\frac{2 \pi i}{D}\left(\frac{\Gamma_{1}}{\Gamma_{1}-i r}\right)^{2}\left\{\begin{array}{c}
\operatorname{trg}\left(t \bar{t} P_{+}\right) \\
-\operatorname{trg}\left(\bar{t} t P_{-}\right)
\end{array}\right\} .
$$

In summary, we obtain for the term $S_{1}(Q)$

$$
\left[S_{1}(Q)\right]_{0} \approx S_{1}(\Lambda)+\frac{4 \pi^{2}}{D^{2}}\left(\frac{\Gamma_{1}}{\Gamma_{1}-i r}\right)^{4} \operatorname{trg}\left(t \bar{t} P_{+}\right) \operatorname{trg}\left(\bar{t} t P_{-}\right)
$$

which is just the first part of Eq. (3.27).

Concerning the term $S_{2}(Q)$, only the linear $\delta Q$-term in (B7) contributes

$$
\begin{aligned}
S_{2}(Q) & =\left(i \frac{\Gamma_{1} D}{2}\right)^{2} \sum_{j} \operatorname{trg}\left[g_{j}^{(0)}(2 \Lambda R) g_{j}^{(0)} P_{+} g_{j}^{(0)}(2 \Lambda R) g_{j}^{(0)} P_{-}\right] \\
& =\left(i \frac{\Gamma_{1} D}{2}\right)^{2} \sum_{j} \operatorname{trg}\left[\left(g_{j}^{(0)}\right)^{2}\left(\bar{g}_{j}^{(0)}\right)^{2}(-4) R P_{+} R P_{-}\right]
\end{aligned}
$$

We can again perform the $H_{0}$-average by using (C7. C8 , 


$$
\left[S_{2}(Q)\right]_{0} \approx\left(i \frac{\Gamma_{1} D}{2}\right)^{2}(-4) \frac{1}{D} \frac{4 \pi}{\left(\Gamma_{1} D-i \omega\right)^{3}} \operatorname{trg}\left[R P_{+} R P_{-}\right]=\frac{4 \pi^{2}}{D^{2}} \frac{\Gamma_{1}^{2}}{\left(\Gamma_{1}-i r\right)^{3}} \operatorname{trg}\left[t P_{+} \bar{t} P_{-}\right]
$$

This expression provides the second part of Eq. (3.27).

The gaussian contractions for (B13) and (B15) can be performed by

$$
\operatorname{trg}\left(t \bar{t} P_{+}\right) \operatorname{trg}\left(\bar{t} t P_{-}\right) \rightarrow \Pi_{D}^{-1} \operatorname{trg}\left[\bar{t} P_{+} t P_{-}\right] \rightarrow \Pi_{D}^{-2} \operatorname{trg}\left(P_{+}\right) \operatorname{trg}\left(P_{-}\right)=\Pi_{D}^{-2},
$$

and

$$
\operatorname{trg}\left[t P_{+} \bar{t} P_{-}\right] \rightarrow \Pi_{D}^{-1} \operatorname{trg}\left(P_{+}\right) \operatorname{trg}\left(P_{-}\right)=\Pi_{D}^{-1},
$$

since $\operatorname{trg}\left(P_{ \pm}\right)=1$. Combining this with $(\overline{\mathrm{B} 13})$ and $(\overline{\mathrm{B} 15})$, we finally obtain the result $(3.28)$.

We mention that this perturbative calculation can be immediately generalized to the orthogonal $(\beta=1)$ and symplectic symmetry classes $(\beta=4)$. In these cases $t$ is a $4 \times 4$-matrix containing $2 \times 2$-blocks $t_{D}$ and $t_{C}$ for the diffuson and cooperon contributions. The result of this generalization just gives the prefactor $2 / \beta$ in $(3.28)$.

\section{APPENDIX C: CONTRIBUTIONS FROM THE FLUCTUATING BREIT-WIGNER WIDTH}

The statistical fluctuations of the Breit-Wigner width $\Gamma$ discussed in subsection IIIE result in further second order contributions when the boundary term $S_{1}(\Lambda)$ is averaged over the diagonal matrix elements. To extract these contributions, we need the following type of simple integrals

$$
I_{n, m}(\gamma)=\lim _{L \rightarrow \infty} \int_{-L}^{L} d y \frac{1}{(y+i \gamma / 2)^{n}} \frac{1}{(y-i \gamma / 2)^{m}} .
$$

Obviously, we have

$$
I_{1,0}=-I_{0,1}=-i \pi \quad, \quad I_{n, 0}=I_{0, n}=0 \quad \text { if } \quad n \geq 2
$$

because for $n \geq 2$ the integration contour can be closed such that there are no singularities inside the contour. For $m=1$, the pol of first order inside the contour results in

$$
I_{n, 1}(\gamma)=(-i)^{n-1} \frac{2 \pi}{\gamma^{n}} \quad, \quad n \geq 1
$$

Furthermore, we obtain by partial integration the recurrence relation

$$
I_{n, m}(\gamma)=-\frac{n}{m-1} I_{n+1, m-1}(\gamma)
$$

which can be solved in terms of the binomial coefficient

$$
I_{n, m}(\gamma)=(-1)^{n-1} i^{n+m-2}\left(\begin{array}{c}
n+m-2 \\
m-1
\end{array}\right) \frac{2 \pi}{\gamma^{n+m-2}} \quad, \quad n, m \geq 1 .
$$

For the subsequent calculation, we need the cases

$$
\begin{aligned}
& I_{1,1}(\gamma)=\frac{2 \pi}{\gamma} \quad, \quad I_{1,2}(\gamma)=-I_{2,1}(\gamma)=i \frac{2 \pi}{\gamma^{2}}, \\
& I_{1,3}(\gamma)=I_{3,1}(\gamma)=-\frac{2 \pi}{\gamma^{3}} \quad, \quad I_{2,2}(\gamma)=2 \frac{2 \pi}{\gamma^{3}} .
\end{aligned}
$$

The moments of the quantities $G_{j}, \bar{G}_{j}$ [see Eq. (3.38)] with respect to the $H_{0}$ average can be expressed in terms of these integrals,

$$
\sum_{j}\left[G_{j}^{n} \bar{G}_{j}^{m}\right]=\frac{1}{D} \lim _{B \rightarrow \infty} \int_{-B / 2}^{B / 2} d H \frac{1}{\left[(x-H)+i\left(D \Gamma_{1}-i \omega\right) / 2\right]^{n}} \frac{1}{\left[(x-H)-i\left(D \Gamma_{1}-i \omega\right) / 2\right]^{m}}=\frac{1}{D} I_{n, m}\left(D \Gamma_{1}-i \omega\right) .
$$


Now, we turn to the average of the boundary term (3.40) which we rewrite as

$$
S_{1}(\Lambda)=\left(B_{0}+B_{1}\right)\left(\bar{B}_{0}+\bar{B}_{1}\right)
$$

with $B_{0}=\sum_{j} G_{j}, B_{1}=-D \delta \Gamma / 2 \sum_{j} G_{j}^{2}$ and analog for $\bar{B}_{0}, \bar{B}_{1}$. The main contribution $\left[B_{0} \bar{B}_{0}\right]_{0}$ has already been considered and evaluated in (3.23). The next contribution is given by [see (3.37)]

$$
\begin{aligned}
{\left[B_{1} \bar{B}_{0}\right]_{0} } & =-\frac{D}{2} \sum_{j, k}\left[\delta \Gamma G_{j}^{2} \bar{G}_{k}\right]_{0}=\frac{D \Gamma_{1}}{2} \sum_{j, k}\left[G_{j}^{2} \bar{G}_{k}\left(\frac{D}{\pi} \sum_{l} G_{l}+i\right)\right]_{0} \\
& =\frac{D \Gamma_{1}}{2} \sum_{j}\left[G_{j}^{2} \bar{G}_{j}\left(\frac{D}{\pi} \sum_{l} G_{l}+i\right)\right]_{0} .
\end{aligned}
$$

The terms with $k \neq j$ vanish since all averages of the type $\left[G_{j}^{n}\right]_{0},\left[\bar{G}_{j}^{n}\right]_{0}$ vanish if $n \geq 2$ [see (C2)]. Furthermore, the contributions with $l \neq j$ cancel for $N \rightarrow \infty$ the second term because $\sum_{l}\left[G_{l}\right]_{0}=I_{1,0}\left(D \Gamma_{1}-i \omega\right) / D=-i \pi / D$. Therefore, by (C7,CE) we obtain

$$
\left[B_{1} \bar{B}_{0}\right]_{0}=\frac{D^{2} \Gamma_{1}}{2 \pi} \sum_{j}\left[G_{j}^{3} \bar{G}_{j}\right]_{0}=\frac{D^{2} \Gamma_{1}}{2 \pi} \frac{1}{D}\left(-\frac{2 \pi}{\left(D \Gamma_{1}-i \omega\right)^{3}}\right)=-\frac{1}{D^{2}} \frac{\Gamma_{1}}{\left(\Gamma_{1}-i r\right)^{3}} .
$$

In an analog way, one finds that $\left[B_{0} \bar{B}_{1}\right]_{0}$ gives the same contribution, such that

$$
\left[B_{1} \bar{B}_{0}\right]_{0}+\left[B_{0} \bar{B}_{1}\right]_{0}=-\frac{2}{D^{2}} \frac{\Gamma_{1}}{\left(\Gamma_{1}-i r\right)^{3}} .
$$

The remaining contribution,

$$
\left[B_{1} \bar{B}_{1}\right]_{0}=\frac{D^{2} \Gamma_{1}^{2}}{4} \sum_{j, k}\left[G_{j}^{2} \bar{G}_{k}^{2}\left(\frac{D}{\pi} \sum_{l} G_{l}+i\right)\left(\frac{D}{\pi} \sum_{m} \bar{G}_{m}-i\right)\right]_{0} \equiv C_{4}+C_{5}+C_{6}
$$

leads to three different subcases with 4,5 , or $6 G$-factors. In the first case only the terms $j=k$ contribute

$$
C_{4}=\frac{D^{2} \Gamma_{1}^{2}}{4} \sum_{j, k}\left[G_{j}^{2} \bar{G}_{k}^{2}\right]_{0}=\frac{D^{2} \Gamma_{1}^{2}}{4} \sum_{j}\left[G_{j}^{2} \bar{G}_{j}^{2}\right]_{0}
$$

For $C_{5}$ and $C_{6}$, we have to consider all different cases of equal or different summations indicies. In the following, we mention only those cases giving a nonvanishing contribution. Actually, for

$$
C_{5}=i \frac{D^{3} \Gamma_{1}^{2}}{4 \pi} \sum_{j, k, m}\left[G_{j}^{2} \bar{G}_{k}^{2}\left(\bar{G}_{m}-G_{m}\right)\right]_{0}
$$

there are two relevant cases:

$$
\begin{aligned}
& C_{5}: j=k \neq m \quad \rightarrow \quad-2 \frac{D^{2} \Gamma_{1}^{2}}{4} \sum_{j}\left[G_{j}^{2} \bar{G}_{j}^{2}\right]_{0}, \\
& C_{5}: j=k=m \quad \rightarrow \quad i \frac{D^{3} \Gamma_{1}^{2}}{4 \pi} \sum_{j}\left[G_{j}^{2} \bar{G}_{j}^{2}\left(\bar{G}_{j}-G_{j}\right)\right]_{0} .
\end{aligned}
$$

The last term

$$
C_{6}=\frac{D^{4} \Gamma_{1}^{2}}{4 \pi^{2}} \sum_{j, k, m, l}\left[G_{j}^{2} \bar{G}_{k}^{2} G_{l} \bar{G}_{m}\right]_{0}
$$

leads to six cases: 


$$
\begin{aligned}
& C_{6}: l \neq(j=k) \neq m \neq l \quad \rightarrow \quad \frac{D^{2} \Gamma_{1}^{2}}{4} \sum_{j}\left[G_{j}^{2} \bar{G}_{j}^{2}\right]_{0} \\
& C_{6}:(j=k) \neq(m=l) \rightarrow \frac{D^{4} \Gamma_{1}^{2}}{4 \pi^{2}} \sum_{j}\left[G_{j}^{2} \bar{G}_{j}^{2}\right]_{0} \sum_{m}\left[G_{m} \bar{G}_{m}\right]_{0} \\
& C_{6}:(j=m) \neq(k=l) \rightarrow \frac{D^{4} \Gamma_{1}^{2}}{4 \pi^{2}} \sum_{j}\left[G_{j}^{2} \bar{G}_{j}\right]_{0} \sum_{k}\left[G_{k} \bar{G}_{k}^{2}\right]_{0} \\
& C_{6}:(j=k=m) \neq l \quad \rightarrow \quad-i \frac{D^{3} \Gamma_{1}^{2}}{4 \pi} \sum_{j}\left[G_{j}^{2} \bar{G}_{j}^{3}\right]_{0} \\
& C_{6}:(j=k=l) \neq m \rightarrow i \frac{D^{3} \Gamma_{1}^{2}}{4 \pi} \sum_{j}\left[G_{j}^{3} \bar{G}_{j}^{2}\right]_{0} \\
& C_{6}: j=k=l=m \rightarrow \frac{D^{4} \Gamma_{1}^{2}}{4 \pi^{2}} \sum_{j}\left[G_{j}^{3} \bar{G}_{j}^{3}\right]_{0} \sim \frac{1}{D} \frac{\Gamma_{1}^{2}}{\left(\Gamma_{1}-i r\right)^{5}} \sim \mathcal{O}\left(\Pi_{D}^{-3}\right) .
\end{aligned}
$$

We observe that (C14), (C16) and (C19) mutually cancel. Furthermore, (C17) is cancelled by $(\mathrm{C} 22)$ and $(\mathrm{C} 23)$. (C24) is already of third order and has to be disregarded. The only second order contributions are (C20) and (C21) which give by $\mathrm{C} 6 \mathrm{C} 8 \mathrm{C}$

$$
\left[B_{1} \bar{B}_{1}\right]_{0}=\frac{D^{4} \Gamma_{1}^{2}}{4 \pi^{2}} \frac{1}{D^{2}} \frac{4 \pi^{2}}{\left(D \Gamma_{1}-i \omega\right)^{4}}[2+i(-i)]=\frac{3}{D^{2}} \frac{\Gamma_{1}^{2}}{\left(\Gamma_{1}-i r\right)^{4}} .
$$

This expression and (C12) are just the extra terms mentioned in (3.41).

${ }^{1}$ M.L. Mehta, Random Matrices, 2nd ed. (Academic Press, New York-London, 1991).

${ }^{2}$ T. Guhr, A. Müller-Groeling, and H.A. Weidenmüller, cond-mat/9707301, Phys. Rep. (in press).

${ }^{3}$ T. Guhr and H.A. Weidenmüller, Ann. Phys. (NY) 193, 472 (1989).

${ }^{4}$ K.B. Efetov, Adv. Phys. 32, 53 (1983).

${ }^{5}$ J.J.M. Verbaarschot, H.A. Weidenmüller and M.R. Zirnbauer, Phys. Rep. 129, 367 (1985).

${ }^{6}$ D.L. Shepelyansky, Phys. Rev. Lett. 73, 2607 (1994).

${ }^{7}$ Y. Imry, Europhys. Lett. 30, 405 (1995).

${ }^{8}$ K. Frahm, A. Müller-Groeling, J.-L. Pichard, and D. Weinmann, Europhys. Lett. 31, 169 (1995);

D. Weinmann, A. Müller-Groeling, J.-L. Pichard, and K. Frahm, Phys. Rev. Lett. 75, 1598 (1995).

${ }^{9}$ F. v. Oppen, T. Wettig, and J. Müller, Phys. Rev. Lett. 76, 491 (1996).

${ }^{10}$ P. Jacquod and D.L. Shepelyansky, Phys. Rev. Lett. 75, 3501 (1995).

${ }^{11}$ Y.V. Fyodorov and A.D. Mirlin, Phys. Rev. B 52, R11580 (1995).

${ }^{12}$ K. Frahm and A. Müller-Groeling, Europhys. Lett. 32, 385 (1995).

${ }^{13}$ D. Weinmann and J.-L. Pichard, Phys. Rev. Lett. 77, 1556 (1996).

14 J.B. French, V.K.B. Kota, A. Pandey and S. Tomsovic, Ann. Phys. (NY) 181, 198 (1988).

${ }^{15}$ F. Leyvraz and T.H. Seligman, J. Phys. A23, 1555 (1990); F. Leyvraz, J. Phys. A26, 6541 (1993).

${ }^{16}$ G. Lenz, Dissertation, Universität Essen, 1992.

17 A. Pandey, Chaos, Solitons and Fractals, Vol. 5, No. 7, 1275 (1995).

18 T. Guhr, Phys. Rev. Lett. 76, 2258 (1996); Ann. Phys. (NY) 250 , 145 (1996).

19 T. Guhr, J. Math. Phys. 32, 336 (1991).

20 T. Guhr and A. Müller-Groeling, J. Math. Phys. 38, 1870 (1997).

${ }^{21}$ A. Altland, M. Janssen, and B. Shapiro, Phys. Rev. E 56, 1471 (1997).

${ }^{22}$ T. Guhr, Supersymmetric Generalization of Dyson's Brownian Motion (Diffusion), to appear in the Proceedings of the NATO ASI workshop on Supersymmetry and Trace Formula: Chaos and Disorder (Cambridge, UK, September 1997).

${ }^{23}$ S. Iida, H.A. Weidenmüller, and J.A. Zuk, Ann. Phys. 200, 219 (1990).

${ }^{24}$ B. L. Al'tshuler and B. I. Shklovskii, Zh. Eksp. Teor. Fiz. 91, 220 (1986) [Sov. Phys. JETP 64, 127 (1986)]. 
Figures
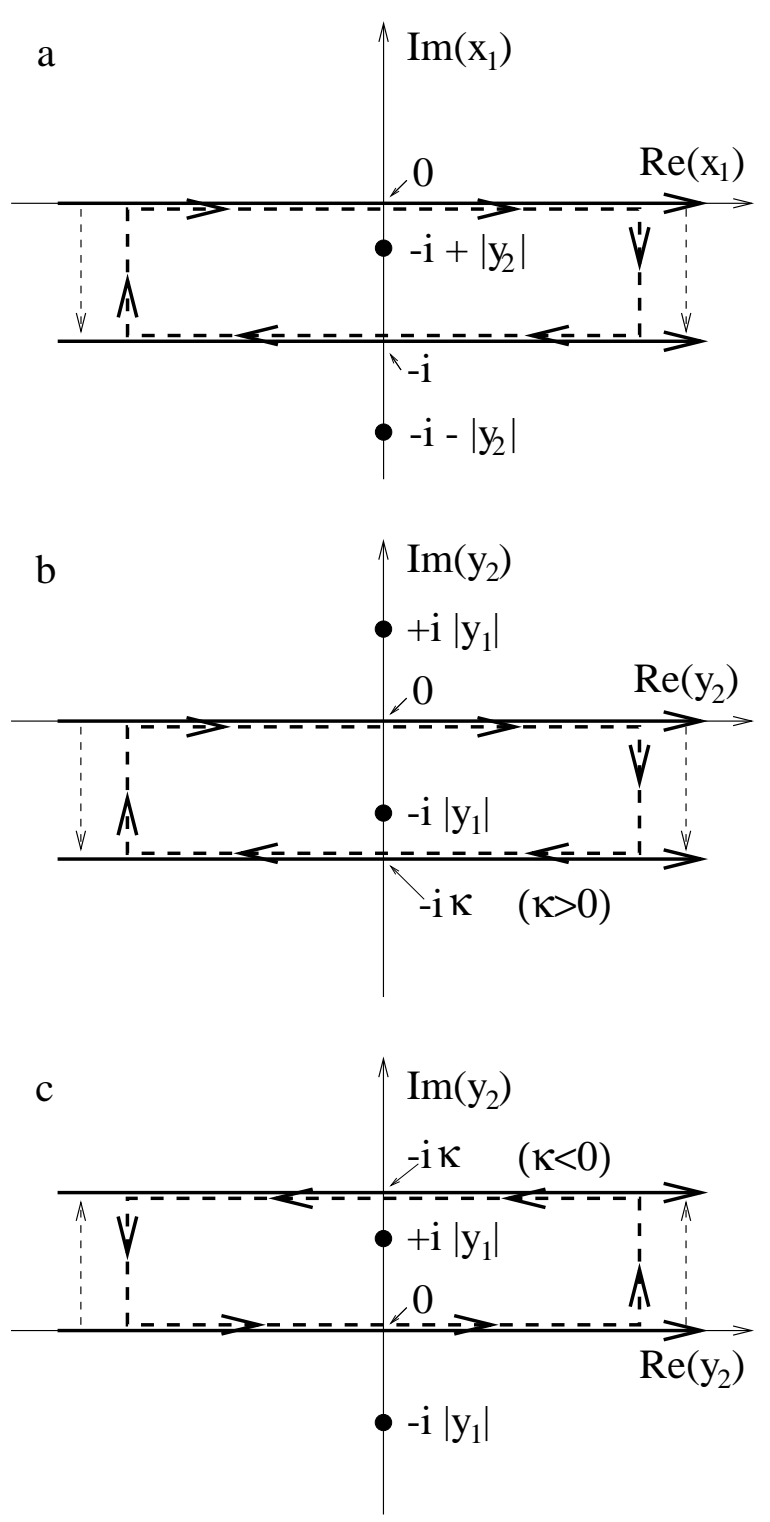

FIG. 1. Graphical illustration of the shifts of the complex integration paths. The two integration paths (full lines) differ by the closed contour integral (dashed line). The direction of the shifts are indicated by the two thin-dashed lines. The first shift $y_{1}=x_{1}+i$ is shown in (a). Here for $\left|y_{2}\right|<1$ the pole $-i+\left|y_{2}\right|$ lies inside the contour and results in the residuum contribution $R_{1}$ [see Eq. (2.17)]. The second shift $u_{2}=y_{2}+i \kappa$ is illustrated in (b) (for $\kappa>0$ ) and (c) (for $\kappa<0$ ). The singularity $\pm i\left|y_{1}\right|$ lies inside the contour if $\left|y_{1}\right|<|\kappa|$. The corresponding residuum contribution is given by $R_{2}$ in Eq. (2.17). 

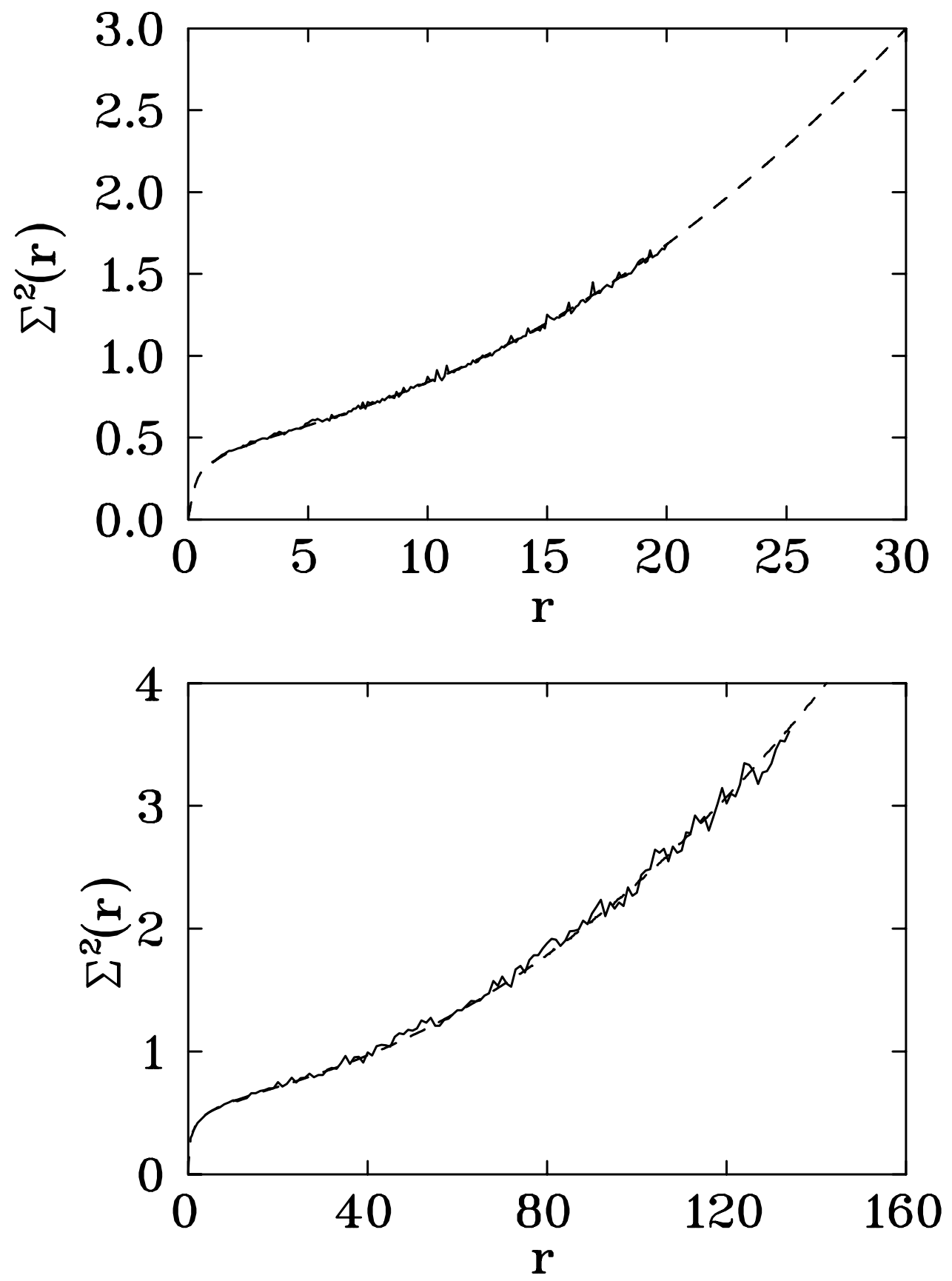

FIG. 2. The number variance $\Sigma^{2}(r)$ as obtained from the numerical simulation (full line) and from our analytical calculation for appropriately chosen $\lambda$ (dashed line). The top and the bottom part of the figure correspond to $\alpha=0.5$ and $\alpha=1.2$ respectively. 


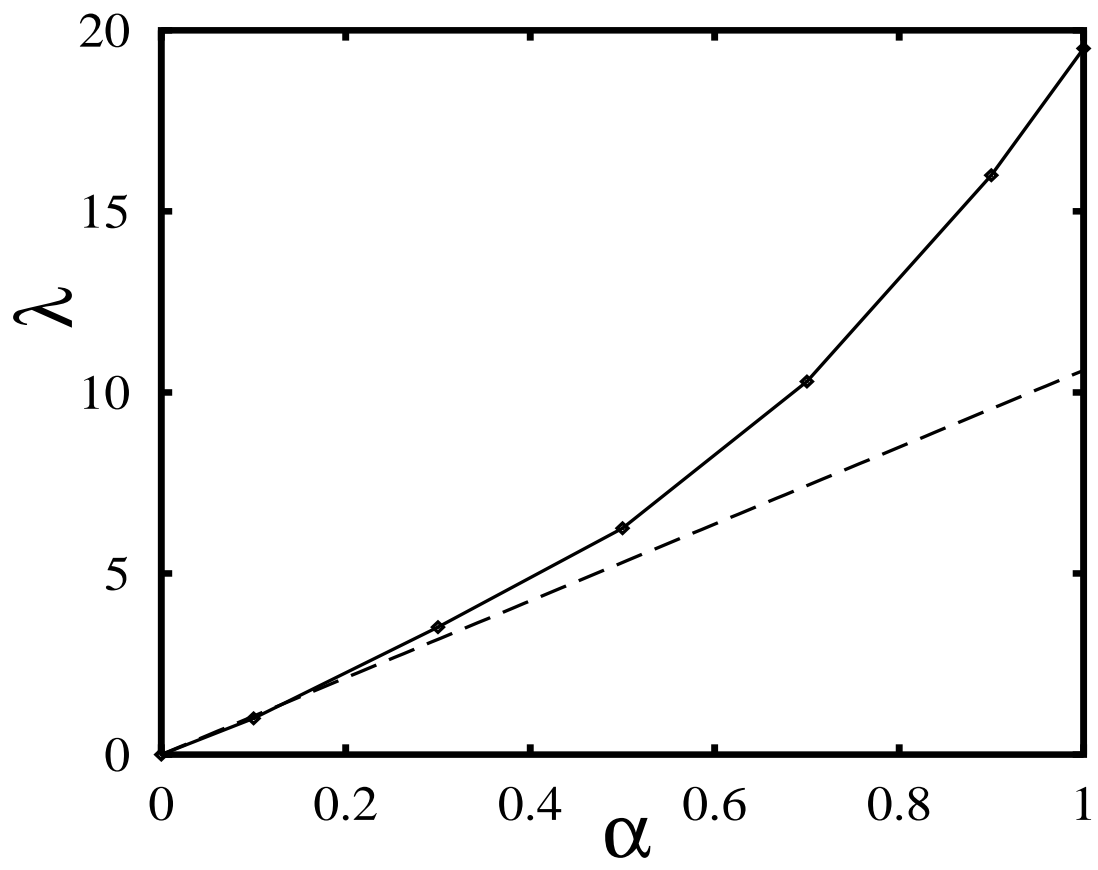

FIG. 3. The parameter $\lambda$ as a function of $\alpha$. Shown is the value of $\lambda$ obtained from the fit of our analytical formula for the number variance to the simulated data (full line and points) and the theoretical expression $\lambda_{\mathrm{th}}=\alpha / D$ (dashed lined). Here $D$ is the mean level spacing for the parameter values mentioned in the text. Note that the critical value $\alpha_{c} \approx 0.95$ corresponds to the situation in which the Breit-Wigner width $\Gamma_{1} D$ equals the radius $R_{0}$ of the unperturbed semicircle density.

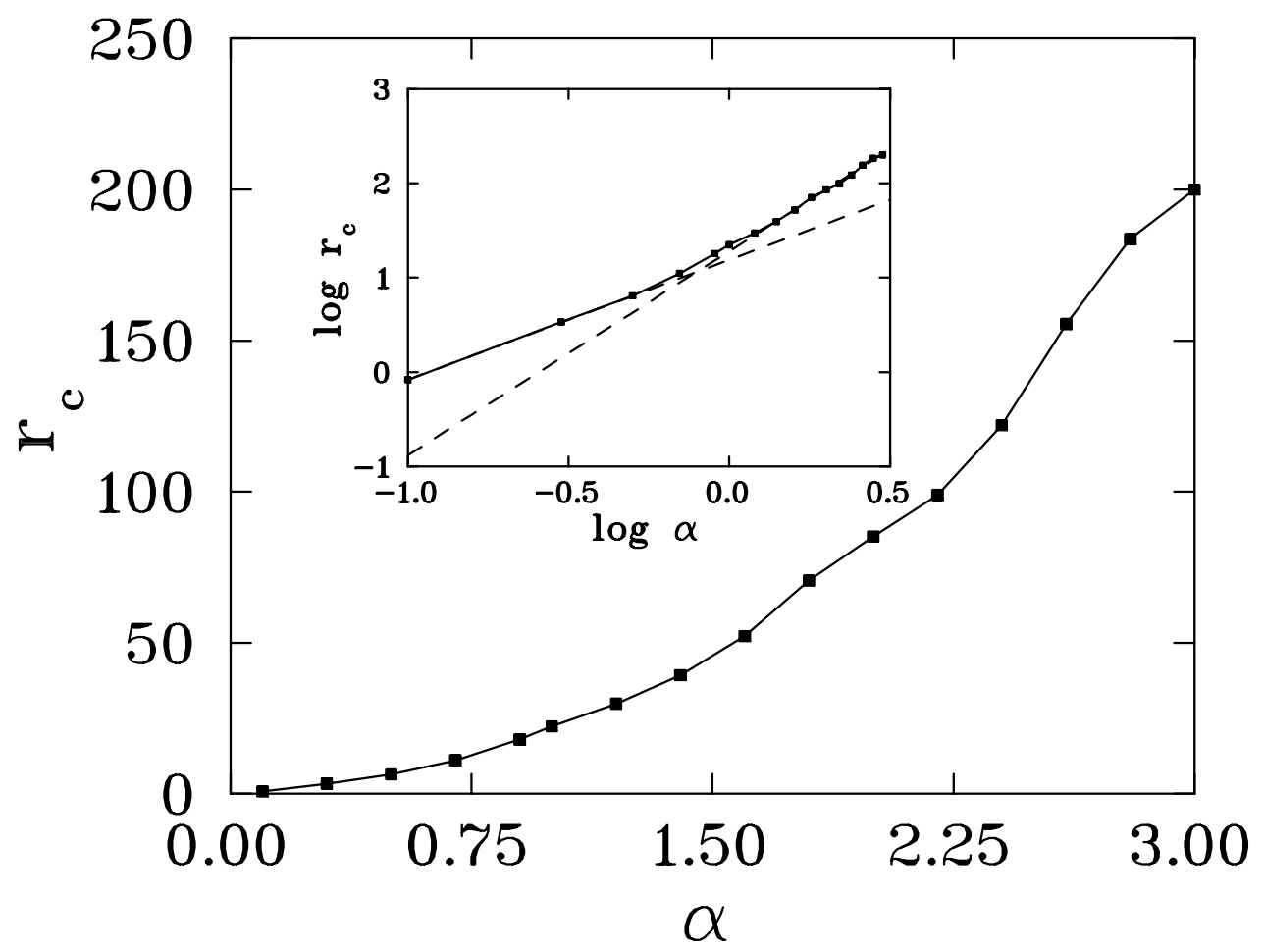

FIG. 4. The dependence of the critical energy $r_{c}$ as defined by the 20 percent criterion explained in the text on the strength parameter $\alpha$. Inset: The same on a logarithmic scale. In addition we have drawn two dashed lines with slopes 1.2 and 2.2 , respectively, to guide the eye. 


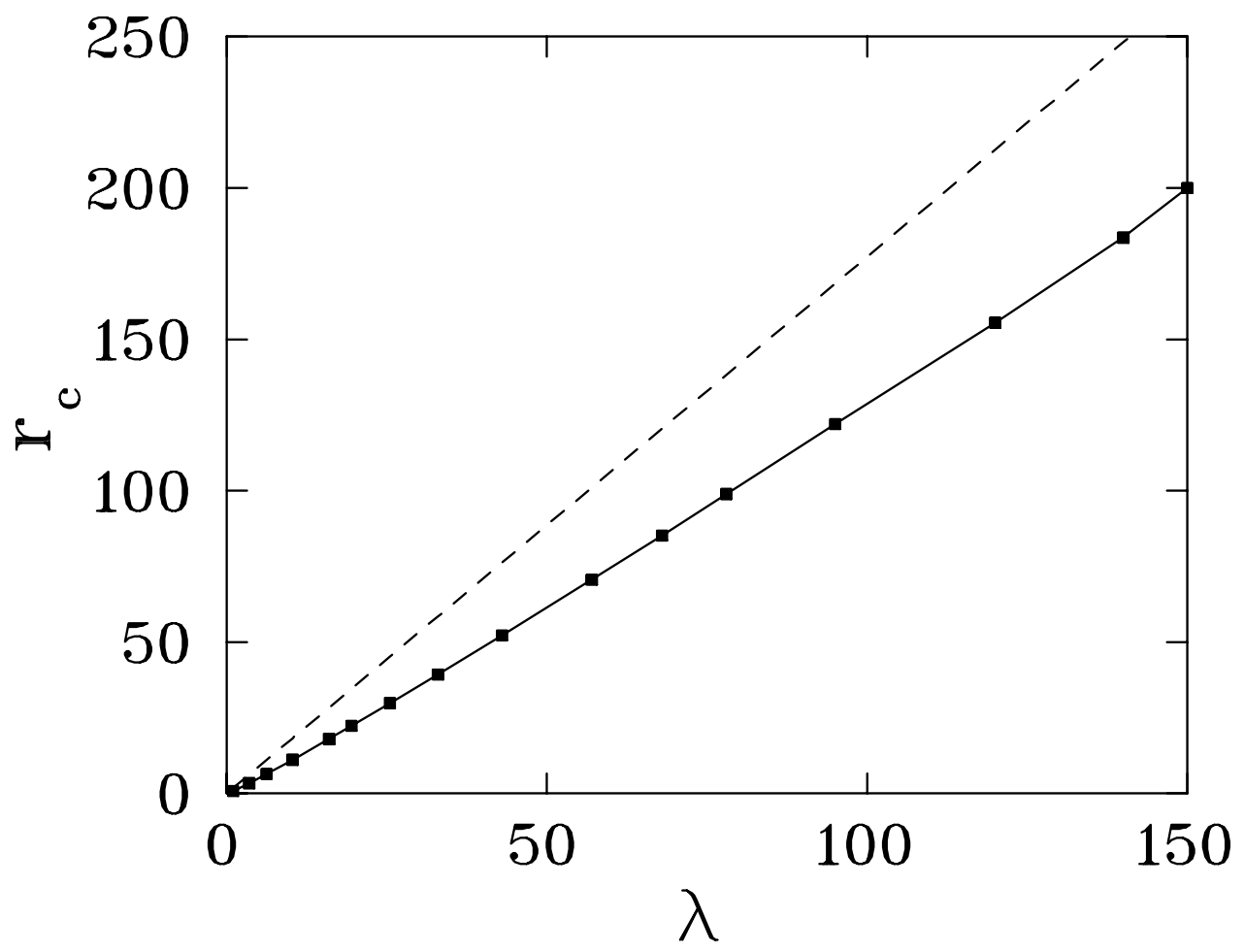

FIG. 5. The dependence of the critical energy $r_{c}$ on the unfolded strength parameter $\lambda$ (full line). The appropriate value of $\lambda$ is obtained from a fit of our analytical formula to the simulated data. For comparison we also show the theoretical energy scale $\sqrt{\Gamma_{1}}=\sqrt{\pi} \lambda$ (dashed line). Both curves are roughly proportional to each other. 